\title{
Proper motion measurements for stars up to 100 kpc with Subaru HSC and SDSS Stripe 82
}

\author{
Tian Qiu ${ }^{1,2 \star}$, Wenting Wang $^{3} \dagger$, Masahiro Takada ${ }^{1}+$, Naoki Yasuda ${ }^{1}$, Željko Ivezić $^{4}$, \\ Robert H. Lupton ${ }^{5}$, Masashi Chiba ${ }^{6}$, Miho Ishigaki ${ }^{6}$, Yutaka Komiyama ${ }^{7}$ \\ ${ }^{1}$ Kavli Institute for the Physics and Mathematics of the Universe (WPI), The University of Tokyo Institutes for Advanced Study (UTIAS), \\ The University of Tokyo, 5-1-5 Kashiwanoha, Kashiwa-shi, Chiba, 277-8583, Japan \\ 2 Department of Physics, The University of Tokyo, 7-3-1 Hongo, Bunkyo-ku, Tokyo 113-0033 Japan \\ ${ }^{3}$ Department of Astronomy, Shanghai Jiao Tong University, Shanghai 200240, China \\ ${ }^{4}$ Department of Astronomy, University of Washington, Box 351580, Seattle, WA 98195, USA \\ 5 Department of Astrophysical Sciences, Princeton University, Peyton Hall, Princeton NJ 08544 USA \\ ${ }^{6}$ Astronomical Institute, Tohoku University, Aoba-ku, Sendai 980-8578, Japan \\ ${ }^{7}$ National Astronomical Observatory of Japan, Mitaka, Tokyo 181-8588, Japan
}

Accepted 2020 December 21. Received 2020 December 21; in original form 2020 April 27

\begin{abstract}
We present proper motion measurements for more than 0.55 million main-sequence stars, by comparing astrometric positions of matched stars between the multi-band imaging datasets from the Hyper Suprime-Cam (HSC) Survey and the SDSS Stripe 82. In doing this we use 3 million galaxies to recalibrate the astrometry and set up a common reference frame between the two catalogues. The exquisite depth and the nearly 12 years of time baseline between HSC and SDSS enable high-precision measurements of statistical proper motions for stars down to $i \simeq 24$. A validation of our method is demonstrated by the agreement with the Gaia

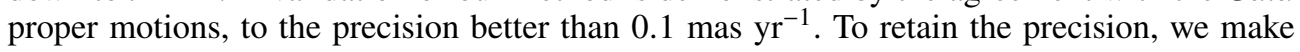
a correction of the subtle effects due to the differential chromatic refraction in the SDSS images based on the comparison with the Gaia proper motions against colour of stars, which is validated using the SDSS spectroscopic quasars. Combining with the photometric distance estimates for individual stars based on the precise HSC photometry, we show a significant detection of the net proper motions for stars in each bin of distance out to $100 \mathrm{kpc}$. The twocomponent tangential velocities after subtracting the apparent motions due to our own motion display rich phase-space structures including a clear signature of the Sagittarius stream in the halo region of distance range $[10,35] \mathrm{kpc}$. We also measure the tangential velocity dispersion in the distance range 5-20 kpc and find that the data are consistent with a constant isotropic dispersion of $80 \pm 10 \mathrm{~km} / \mathrm{s}$. More distant stars appear to have random motions with respect to the Galactic centre on average.
\end{abstract}

Key words: proper motions - methods: statistical - Galaxy: kinematics and dynamics

\section{INTRODUCTION}

Our Milky Way (MW) Galaxy provides a wealth of information on the physics of galaxy formation and the underlying cosmology (e.g. Moore et al. 1999; Klypin et al. 1999; Boylan-Kolchin et al. 2011; Cautun et al. 2015; Helmi et al. 2018; Laporte et al. 2018; Gallart et al. 2019; Bonaca et al. 2019). The information can be revealed from the positions, velocities and chemistry of stars in the Galaxy, and from its satellites, globular clusters and tidal streams, which give us very powerful diagnostics to probe kinematical structures

\footnotetext{
$\star$ Contact e-mail: tian.qiu@ipmu.jp

$†$ Contact e-mail: wenting.wang@sjtu.edu.cn

† Contact e-mail: masahiro.takada@ipmu.jp
}

of the MW halo as well as the assembly history of the MW in phase space. The readers can see Helmi (2020) for a review.

Among the 6-dimensional phase space information, proper motions of stars in the MW is most expensive to be measured precisely, especially for faint stars out to large distances. It usually requires long time baselines, accurate astrometry and careful corrections of systematic errors. However, full phase-space information is very informative for proper scientific inferences. For example, if the velocity anisotropy of stars cannot be directly inferred from data due to unavailable proper motions, the MW mass is often estimated with large systematic uncertainties, which is the so-called "mass-anisotropy" degeneracy (e.g Battaglia et al. 2005; Wang et al. 2019).

Proper motions can be measured by comparing the astrometric 
positions of stars observed over different years. Early proper motion surveys rely on multiple exposures taken at different times of the same survey, which involve the nearly all-sky but shallow surveys (e.g. Luyten \& Hughes 1980; Høg et al. 2000; Zacharias et al. 2000; Monet et al. 2003), and the deeper surveys covering smaller areas (e.g. Hambly et al. 2001a,b; Ruiz et al. 2001; Lépine et al. 2003). Later on, with new wide and deeper surveys such as the Sloan Digital Sky Survey (hereafter SDSS; York et al. 2000), proper motions of stars can be further measured by comparing their astrometry in two or multiple surveys spanning even longer time baselines (e.g. Gould \& Kollmeier 2004; Munn et al. 2004; Koposov et al. 2013; Munn et al. 2014; Tian et al. 2017; de Boer et al. 2018).

Since proper motions are measured by comparing the astrometric positions, any systematic errors in astrometry can lead to systematics in measured proper motions. The most significant error is due to the different reference frames for observations conducted at different times, which is caused mainly due to the motion of astrometric reference stars. This is often calibrated against distant quasars or galaxies, which have zero proper motions, given a limited accuracy due to statistical errors. Thus apparent "proper motions" of galaxies or quasars, if observed, reflect the underlying systematics. For example, Gould \& Kollmeier (2004) used quasars to correct for proper motions in SDSS/USNO-A and USNO-B sources. Munn et al. (2004) further provided an improved proper motion catalogue to Gould \& Kollmeier (2004). The USNO-B positions were recalibrated using SDSS galaxies. The catalogue is $90 \%$ complete down

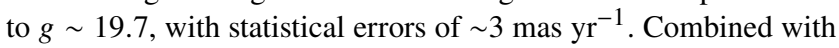
photometric parallax and metallicity (Jurić et al. 2008; Ivezić et al. 2008), this catalogue was used to study the kinematics of stars in a distance range from $100 \mathrm{pc}$ to $\sim 13 \mathrm{kpc}$ (Bond et al. 2010).

More recently, Koposov et al. (2013) used positional offsets between stars and quasars at multiple epochs over 7 years from the SDSS Stripe 82 data (Annis et al. 2014), and then measure statistical proper motions for stars at $r \lesssim 21$ in the Sagittarius stream, with the precision of $\sim 0.1$ mas $\mathrm{yr}^{-1}$. Munn et al. (2014) provided deeper proper motion measurements by matching objects in SDSS DR7 to two independent observations, each covering more than $1000 \mathrm{deg}^{2}$ down to $r=22$ and $r=20.9$ respectively. Systematic errors were

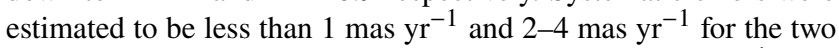
observations, with typical statistical errors of 5 to $15 \mathrm{mas} \mathrm{yr}^{-1}$ from the bright to faint end.

Moreover, by combining 2MASS, SDSS, Pan-STARRS1 and Gaia DR1 astrometry, the Gaia-PS1-SDSS (hereafter GPS1) proper motion catalogue (Tian et al. 2017) contains 350 million sources down to $r \sim 20$, which covers nearly $3 / 4$ of the sky. Tian et al. (2017) used galaxies to set up a reference frame and bring different observations to this frame. The typical systematic and statistical er-

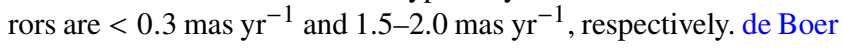
et al. (2018) studied the proper motions across the Galactic anticentre region using recalibrated SDSS astrometry in combination with positions from Gaia DR1.

Gaia is producing proper motion measurements with unprecedented high precisions. The Gaia second data release (DR2) provides position, parallax and proper motion solutions for about 1.3 billion objects (Gaia Collaboration et al. 2018). The proper motion

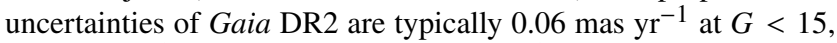
$0.2 \mathrm{mas} \mathrm{yr}^{-1}$ at $G=17$ and $1.2 \mathrm{mas} \mathrm{yr}^{-1}$ at $G=20$ (Gaia Collaboration et al. 2016, 2018). The precision is expected to further improve with the increased duration by future Gaia observations.

The proper motion measurements/catalogues introduced above, however, are still relatively shallow. For example, the Gaia proper motions are mainly useful for science of nearby main- sequence stars (distances up to a few $\mathrm{kpc}$ ) and rare populations of distant, luminous stars such as RR-Lyrae stars and K-giants (distances up to at most a few tenth of $\mathrm{kpc}$ ). The upcoming Rubin Observatory's LSST (Jurić et al. 2017; Ivezić et al. 2019, Legacy Survey of Space and Time) survey is able to measure proper motions for a sample of $\sim 200$ million F/G main-sequence stars out to a distance of $\sim 100 \mathrm{kpc}$. LSST is designed to be able to image $\sim 10,000$ square degrees of the southern sky per night, repeatedly over 10year operation (Ivezić et al. 2019; LSST Science Collaboration et al. 2017).

In this paper, we use the ongoing, deep Hyper Suprime-Cam Subaru Strategic Program Survey (Aihara et al. 2018a, hereafter HSC-SSP or HSC) to achieve precise measurements of statistical proper motions for main-sequence stars down to much fainter magnitudes $i \simeq 24$ : we compare the HSC astrometry with the deep SDSS Stripe 82 data, for the overlapping region of about $100 \mathrm{deg}^{2}$. In many aspects, HSC is a pre-implemented version for the future LSST survey, with similar depth and data quality. In our analysis, instead of using sources detected on single exposure images, we compare the astrometry of sources detected on coadd images in both HSC and SDSS, which enables us to push down to $r \sim 24$, i.e., 2 to 4 magnitudes fainter than previous proper motion catalogues ${ }^{1}$. However, though HSC and SDSS Stripe 82 data are on average separated by 12 years, the SDSS Stripe 82 spans 7-8 years of observing duration. Thus, we take into account the mean epoch for individual stars taken from each of the HSC and Stripe 82 coadd images, by averaging all input exposure images. The difference between HSC and Stripe 82 mean epochs is then adopted as the time baseline for the proper motion measurements of individual stars.

In our analysis, we also use galaxies to do astrometry recalibration and bring the reference frames between HSC and SDSS Stripe 82 to be the same. Photometric distances/parallaxes are estimated for individual stars based on the precise HSC multi-band photometry (Jurić et al. 2008; Ivezić et al. 2008), which enable us to measure the tangential velocity as a function of the photometric distance. We validate our proper motion measurements by comparing with the Gaia DR2 proper motions for the matched stars. We construct judiciously selected sub-samples of stars to harness the power of statistics and present the first significant detection of proper motions for main-sequence stars at distances up to $100 \mathrm{kpc}$.

This paper is structured as follows. We introduce the surveys and corresponding source catalogues in Section 2, including stars and galaxies of HSC and SDSS Stripe 82, Gaia DR2 stars and SDSS DR14 quasars. In Section 3 we describe our methods of matching sources between different surveys, calibration of systematic errors and calculation of photometric distances. We present detailed results based on the measured proper motions and the comparisons/validations through Gaia proper motions in Section 4. In Section 5 we discuss an implication of our proper motion measurements for the Sagittarius stream, and then discuss possible systematic and statistical uncertainties inherent in our measurements. We conclude in the end (Section 6). In Appendix, we also give details of our analysis. 


\begin{tabular}{lr}
\hline \hline Type & Number of objects \\
\hline HSC total $\left(i_{\mathrm{HSC}}<24.5\right)$ & 8273848 \\
S82 total $\left(i_{\mathrm{S} 82}<24.2\right)$ & 4841985 \\
\hline Matched total & 4302686 \\
Galaxies for recalibration & 3153981 \\
Quasars & 8757 \\
Stars for proper motion measurements & 555529 \\
Blue stars & 97087 \\
Stars matched with Gaia & 117703 \\
\hline
\end{tabular}

Table 1. Number of objects used in this paper. "HSC total" denotes the total number of detected objects with $i_{\mathrm{HSC}}<24.5$ in the HSC data and in the overlapping footprints of HSC and S82 (about $100 \mathrm{deg}^{2}$ ). "S82 total" denotes the total number of detected objects with $i_{\mathrm{S} 82}<24.2$ in the S82 data in the overlapping footprints. Note, however, that the overlapping footprints of HSC and S82 are not exactly the same due to the difference in masked regions (e.g. around bright stars). These are our master samples of objects. Other rows denote the number of different objects: the matched objects ("Matched total"), the matched galaxies ("Galaxies for recalibration"), the matched quasars with the SDSS DR14 spectroscopically-confirmed quasars in the S82 region ("Quasars"), the stars used for proper motion measurements, the "blue" stars with $g_{\mathrm{PSF}}-r_{\mathrm{PSF}}<0.6$ among the matched stars, and the number of matched stars with the Gaia catalogue among the matched stars of HSC and S82.

\section{DATA}

In this section we describe details of data/catalogues, the Hyper Suprime-Cam and the SDSS that we use in this paper. We summarise the number of objects used in this paper in Table 1.

\subsection{SDSS Stripe 82 (S82)}

The Sloan Digital Sky Survey (SDSS) used a dedicated wide-field $2.5 \mathrm{~m}$ telescope (York et al. 2000; Gunn et al. 2006) to image the sky in drift-scanning mode with five optical filters, ugriz (Fukugita et al. 1996; Gunn et al. 1998). The effective single exposure time per filter is 54.1 seconds. For stellar sources, the 50\% completeness limits are estimated as $u, g, r, i, z \simeq 22.5,23.2,22.6,21.9,20.8$, respectively (Abazajian et al. 2003).

The SDSS imaged a $275 \mathrm{deg}^{2}$ region, multiple times, in the Celestial Equatorial region, so-called Stripe 82 (hereafter we call the data in the SDSS Stripe 82 region for short as S82). The coadd images in ugriz are $\sim 1.5$ magnitudes deeper than those in the SDSS single-pass data (Annis et al. 2014; Jiang et al. 2014). The region is $2.5^{\circ}$ wide along Dec. and traces the Celestial Equator in the South Galactic Cap, more precisely the contiguous region in the ranges of $-50^{\circ} \leq$ R.A. $\leq 60^{\circ}$ and $-1.25^{\circ} \leq$ Dec. $\leq 1.25^{\circ}$. The publicly-available data and catalogues of S 82 are constructed from the coadds of $\sim 20$ multiple images, taken from 1998 September to 2005 November. The median seeing is $1.1^{\prime \prime}$ and the $50 \%$ completeness limits are $r=23.5$ for galaxies and $r=24.3$ for stars, respectively.

In this study we download the data from CasJobs, with the detailed sample selection query provided in Appendix A. About $100 \mathrm{deg}^{2}$ of the S82 footprint overlaps with HSC as we describe

1 The light-motion catalogue in the SDSS Stripe 82 region is available (Bramich et al. 2008), which is complete down to $r \sim 21.5$. Proper motions are derived by recalibrating the astrometry against some mid-run data. In this paper, we are not directly using these proper motions in our study. below, which gives about 5 million objects with $r<24.2$ in total. This is the master S82 catalogue. However, as we will show later, the purity of star catalogues in S82 is low for stars at $i \gtrsim 21$, due to relatively poor seeing conditions compared to HSC.

For the positions of stars/galaxies/quasars, we use their centroid positions in $r$-band as our fiducial choice. We will also use the centroid positions of the other filters to test possible effects of systematic errors on our results.

\subsection{Subaru HSC}

The Hyper Suprime-Cam (HSC) camera at the prime focus of the $8.2 \mathrm{~m}$ Subaru Telescope is a wide-field imaging camera with $1.77 \mathrm{deg}^{2}$ field-of-view (Miyazaki et al. 2018; Komiyama et al. 2018). The HSC survey is using about 330 nights of Subaru time, since 2014, to conduct a multi-band wide-field imaging survey (Aihara et al. 2018a). The HSC data we use in this paper is taken from the ongoing HSC-Wide survey, which is a part of the three layers with different combinations of depth and area, and aims at covering about $1,400 \mathrm{deg}^{2}$ of the sky with five filters, grizy, with $5 \sigma$ point-source depths of $(g, r, i, z, y) \simeq(26.5,26.1,25.9,25.1,24.4)$ measured in $2 "$ aperture (Kawanomoto et al. 2018; Furusawa et al. 2018). The total exposure times range from $10 \mathrm{~min}$ in the $g$ - and $r$-bands to $20 \mathrm{~min}$ in the $i$-, $z-$, and $y$-bands, divided into individual exposures of $\sim 3$ min each.

Since $i$-band images are used for galaxy shape measurements in weak lensing analysis, $i$-band images are preferentially taken when the seeing is better (Mandelbaum et al. 2018). The HSC $i$ band images have a median PSF FWHM of $0.6^{\prime \prime}$. The superb image quality brings benefits for our study because the HSC images allow for a robust selection of stars or equivalently a less contamination of galaxies to the star catalogue as we will show below. The details of the software used to reduce the data are given in Bosch et al. (2018) (also see Furusawa et al. 2018). The fundamental calibration of photometry and astrometry was done against the public PanSTARRS catalogue (Schlafly et al. 2012; Tonry et al. 2012; Magnier et al. 2013; Chambers et al. 2016; Magnier et al. 2016).

In this paper we use the primary photometric sources with $i_{\mathrm{HSC}}<24.5$ from the S18a internal data release, which is almost identical to the second public data release (Aihara et al. 2019). We have about 8.3 million objects as the master HSC catalogue. We adopt the data in the HSC-Wide survey regions that have the 5filter data and the approximately full depths, in the S82 region. We provide the sample selection query in Appendix A.

For the positions of stars/galaxies/quasars, we use their centroid positions in $i$-band as our fiducial choice for the HSC data.

\subsection{Other datasets}

We also use Gaia $^{2}$ DR2 (Gaia Collaboration et al. 2018) and SDSS DR14 quasars (Pâris et al. 2018) for validation and calibration of our proper motion measurements, as we will describe later. The numbers of DR14 quasars and Gaia stars, used in this paper, are given in Table 1.

\footnotetext{
2 https://www.cosmos.esa.int/web/gaia/data-release-2
} 


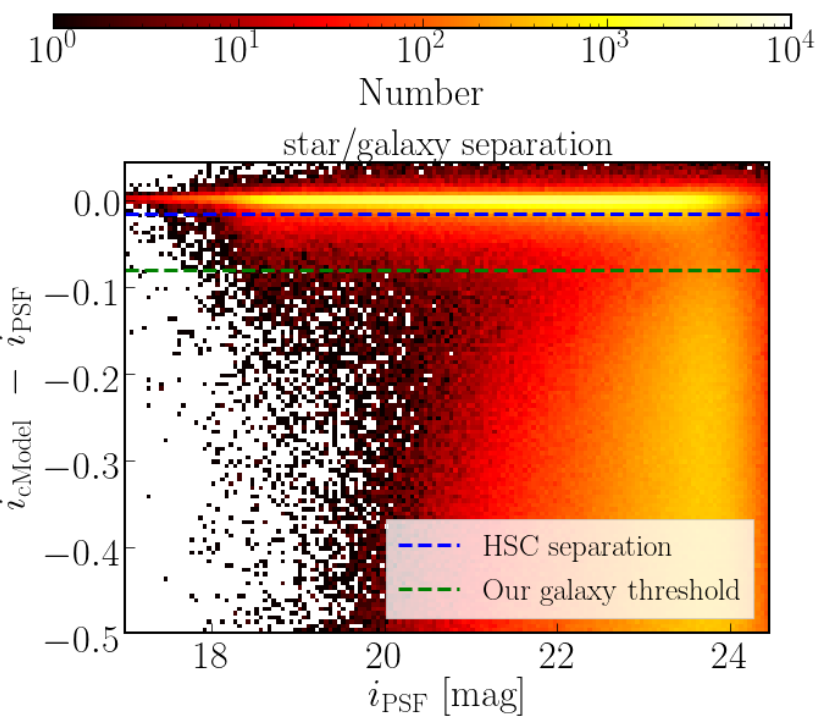

Figure 1. The number distribution of all objects in terms of HSC $i$-band PSF magnitudes ( $x$-axis) versus HSC $i$-band cModel minus PSF magnitudes ( $y$-axis). Colour denotes the number of objects in each grid of the two dimensional space, as denoted by the upper colour bar. We select stars with the cut of $i_{\mathrm{HSC}, \mathrm{cModel}}-i_{\mathrm{HSC}, \mathrm{PSF}}>-0.015$ (dark blue dashed line). To ensure a pure sample of galaxies, we adopt a cut of $i_{\mathrm{cModel}}-i_{\mathrm{HSC}, \mathrm{PSF}}<-0.08$ (green dashed line).

\section{METHODOLOGY}

In the following, we describe how to classify photometric sources as stars or galaxies, and how we can further improve the classification to reduce the contamination. After star/galaxy separation, we identify the same stars/galaxies in the HSC and S82 catalogues, by matching the positions of stars/galaxies between the two catalogues. We will also introduce our approach of using galaxies to correct for systematics in astrometric solutions of the two catalogues.

\subsection{Star/Galaxy separation}

Both HSC and S82 have their own criteria for star/galaxy separation. For both data, a point source is identified on the basis of the ratio between PSF and cModel fluxes (Abazajian et al. 2004). For HSC, $i$-band measurements have the best seeing so that we adopt the $i$ band "extendedness" parameter for the classification. We adopt the criterion $i_{\mathrm{HSC}, \mathrm{cModel}}-i_{\mathrm{HSC}, \mathrm{PSF}}>-0.015$ to define point sources.

For S82, $r$-band has the best resolution. Point sources are defined from objects satisfying $\left|r_{\mathrm{S} 82, \text { model }^{-}}{ }_{\mathrm{S} 82, \mathrm{PSF}}\right| \leq 0.03$, which is much more stringent than the threshold $(0.145)$ adopted for the nominal SDSS depth thanks to the deeper depth of SDSS S82.

As we will show later, we will also use a catalogue of galaxies to correct for systematic errors in astrometric solutions of stars between the HSC and S82 catalogues. To define a secure catalogue of galaxies, we use the HSC catalogue and define, as galaxies, objects satisfying the condition of $i_{\mathrm{HSC}, \mathrm{cModel}}-i_{\mathrm{HSC}, \mathrm{PSF}}<-0.08$. The threshold value of -0.08 corresponds to the position of a dip in Figure 1, which is determined empirically. We believe that the criterion can give a more stringent selection of galaxies because the threshold is sufficiently far from the threshold of star separation, $i_{\mathrm{HSC}, \mathrm{cModel}}-i_{\mathrm{HSC}, \mathrm{PSF}}>-0.015$, and photometric errors are un-
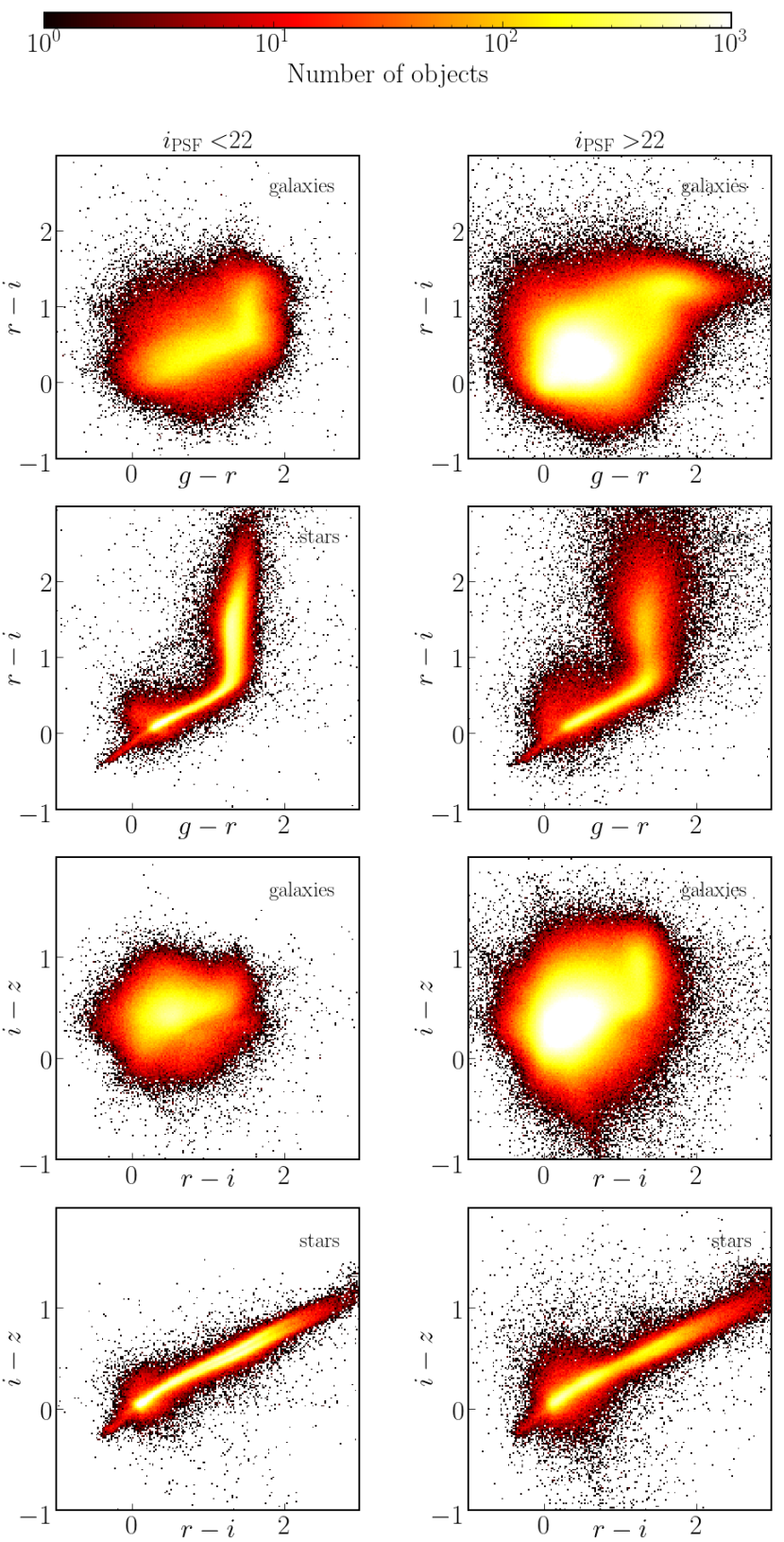

Figure 2. The colour-colour diagrams of stars and galaxies as denoted by the label in each panel. Here colour in each grid of panels represents the number of objects in the grid, as denoted by the upper colour bar. To calculate colour for each object, we use the HSC cModel and PSF magnitudes for galaxies and stars, respectively. The left-column panels show the results for bright objects with $i_{\mathrm{HSC}, \mathrm{PSF}}<22$, while the right panels show the results for faint objects $\left(i_{\mathrm{HSC}, \mathrm{PSF}}>22\right)$. There is no obvious signs of galaxy contamination around stellar locus.

likely to cause a scatter of stars into this galaxy region thanks to the deep HSC photometry.

In Figure 2 we show the distributions of stars and galaxies in the colour-colour plane, $(g-r)$ vs. $(r-i)$ and $(r-i)$ vs. $(i-z)$, for the bright and faint samples that are divided based on the HSC $i$-band apparent magnitudes, $i_{\mathrm{HSC}, \mathrm{PSF}}<22$ or $>22$, respectively. Stars are distributed along a narrower locus in each colour space, while galaxies display a broader distribution reflecting the diversity 
of galaxy colours, partly due to their shifted colours with redshifts. We can use the colour information to define a more secure sample of galaxies, e.g. by using extended objects that are sufficiently away from the stellar locus. Then we will use such a sample to check whether our recalibration of astrometry using galaxies are robust against different galaxy samples (see Section 3.3.1 for details).

The stellar locus in Figure 2 displays a broadened feature at some colour. These are either non-main-sequence stars, or caused by failures in photometry (e.g. Jurić et al. 2008). As we will introduce in Section 3.4, we calculate photometric distances for main-sequence stars based on their photometric colour. To ensure accurate colour measurements and also guarantee a minimal amount of contamination by non-main-sequence stars in our measurements, we choose to exclude those stars (or star-like objects) that are away from the stellar locus for our analysis throughout this paper. Explicitly, we divide our sample of stars into different bins according to their $g-i$ colour, and for each bin, we make 3- $\sigma$ clipping based on their $g-r$ colours. This clipping discards about 47,000 objects (discards about 12,000 objects from the catalogue of blue stars which is the sample for our results beyond $30 \mathrm{kpc}$ ). Here we define the "blue" stars as those stars with colour $g-r<0.6$, which are probing distant halo stars rather than younger disk stars, following Ivezić et al. (2008). In fact, they are closer in colour to yellow stars (like our Sun). However, we still call them "blue" stars throughout this paper since they are at the blue end of colour range probed by our data, which are also used to differentiate from "red" stars whose colour $g-r$ larger than 0.6. Besides, we discard those extremely blue stars whose $g-r$ or $g-i$ colour less than 0.2 to avoid the contamination of white dwarfs, as we will discuss in Section 5.4 in detail. This cut is also desired because the photometric distance estimation also requires a $g-i$ colour limitation from 0.2 to 4.0 as given by Eq. (A7) in Jurić et al. (2008). This is a minor fraction compared to the number of remaining stars used in the following analysis as can be found from Table 1.

\subsection{Methodology of proper motion measurements}

\subsubsection{Matching objects in the HSC and S82 catalogues}

To measure proper motions of stars from the HSC and S82 data, it is important to construct a matched catalogue of stars between the two datasets. Here we describe our method to define the matched catalogues of stars, galaxies and quasars.

The catalogues of objects we use in this paper are constructed based on the coadd images of HSC and S82. The coadd images are contributed by multiple single exposures. For HSC, all single exposures overlapped with a particular "tract" are resampled to an output plane centred on the tract. They are then weighted-averaged to produce the coadd image (Bosch et al. 2018; Aihara et al. 2018b, 2019). For S82, images from each single run were resampled and coadded following a similar approach (Annis et al. 2014). For both HSC and S82, objects are detected, deblended and measured from the coadd images.

For HSC, detections are at first made for each band, with the detected footprints and peaks of sources in different bands afterwards merged to ensure consistency across different bands ${ }^{3}$. These peaks are deblended and a full suite of source measurement algorithm is run on all objects, yielding independent measurements of

3 Also see https://www . astro.princeton. edu/ ${ }^{\sim}$ rhl/photo-lite. pdf.

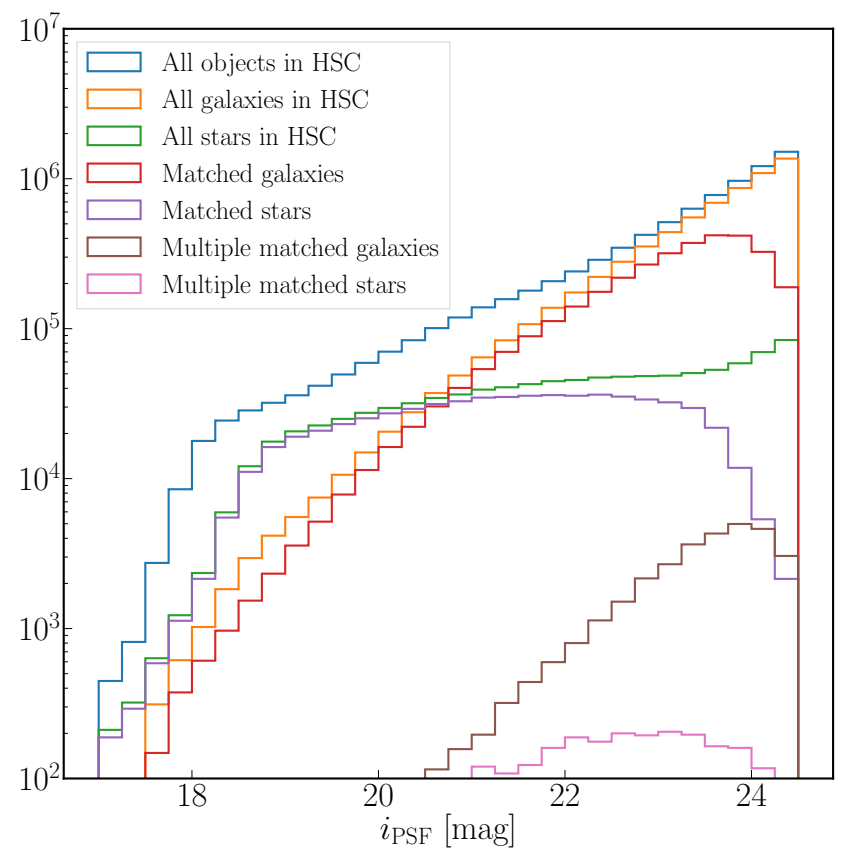

Figure 3. The number of objects against the HSC $i$-band PSF magnitudes. The blue histogram shows the distribution for all detected HSC objects in the overlapping footprints between HSC and S82. The orange and green histograms show the distributions for the HSC-detected galaxies and stars, respectively. In comparison, the red and purple histograms show the distributions for the matched galaxies and stars of HSC and S82 catalogues (see text for details). The brown and magenta histograms denote the galaxies and stars, respectively, where those in HSC catalogue are matched with multiple objects in $\mathrm{S} 82$.

positions and source parameters in each band. A reference band of detection is then defined for each object based on both the signalto-noise and the purpose of maximising the number of objects in the reference band. Finally, the measurement of sources is run again with the position and shape parameters fixed to the values from the measurement in the reference band, which is usually the HSC $i$-band given its best image quality (Bosch et al. 2018; Mandelbaum et al. 2018).

For S82, a detailed description about its astrometric calibration can be found in Pier et al. (2003). SDSS $r$-band photometric CCDs are served as the astrometric reference CCDs, which means SDSS object positions are based on the $r$-band centroid measurement.

We construct a catalogue of matched objects by searching for the same objects in the HSC and S82 catalogues with their positions. To do this we use the master catalogues of objects selected with magnitude cuts, $i_{\mathrm{HSC}}<24.5$ and $i_{\mathrm{S} 82}<24.2$, for HSC and $\mathrm{S} 82$ respectively. The reason that we stop at $i_{\mathrm{S} 82}=24.2$ for $\mathrm{S} 82$ is because the photometric errors increase very quickly beyond $i_{\text {S82 }} \simeq 24$.2. For the matching, we start with each object in the HSC catalogue and then search for a counterpart object(s) in the S82 catalogue within an angular separation of $1^{\prime \prime}$ in radius for the HSC object. The search region of $1^{\prime \prime}$ radius is chosen because stars with transverse velocity of $220 \mathrm{~km} / \mathrm{s}$ at $1 \mathrm{kpc}$ leads to an angular offset of about $0.5^{\prime \prime}$ or 500 mas over a 10 -year baseline, corresponding to a

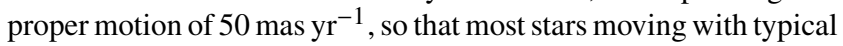
moderate velocities can be matched properly with the search region. Since we are mainly interested in distant stars up to $\sim 100 \mathrm{kpc}$, the 
choice of 1 " radius is safe, even if those stars have larger velocities. The depth and superb image quality of HSC help construct a secure catalogue of matched objects, especially stars, down to $i_{\mathrm{HSC}} \simeq 24.5$.

Figure 3 shows the matching results. We have about 4.3 million matched objects in total (see the blue histogram). Among these, there are about 0.55 million objects which are classified as stars in HSC and 3.2 million objects which are classified as galaxies in both catalogues, as given in Table 1. Note that we have excluded stellar or star-like objects away from the stellar locus (see Section 3.1), and the fraction of contamination by non-main-sequence stars such as giants in our sample would be at most 5\% (Jurić et al. 2008).

The sum of "Galaxies for recalibration" and "Stars for proper motion measurements" in Table 1 is smaller than the total number of matched objects. This is because some of the HSC galaxies are matched to objects classified as stars in S82, and we have discarded these objects from our sample of galaxies. In addition, there is a small fraction of objects which are not classified as either galaxies or stars.

In this paper, the catalogue of stars is the most fundamental ingredient for our study of proper motion measurements, so we first focus on the results for stars. Comparing the green and purple histograms in Figure 3, most of HSC stars with $i_{\mathrm{HSC}, \mathrm{PSF}}<22$ have a matched counterpart in S82; more precisely, about $92 \%$ of HSC stars have the matched S82 object. However, about $8 \%$ of HSC stars are unmatched. We checked that this is mainly because HSC and S82 adopt different masks (mainly due to bright stars) in the footprints ${ }^{4}$. For fainter stars at $i>22$, the matching performance starts to degrade. About 80, 70, and 20\% of HSC stars at $i_{\mathrm{HSC}} \simeq 22,23$ and 24 have matched objects in S82, respectively, and the matching drops below $10 \%$ at $i_{\mathrm{HSC}} \simeq 24.2$, the limit of object detection for S82.

We should note that some HSC stars are matched only to "galaxies" in the S82 catalogue, which occurs due to the relatively poor seeing condition of SDSS (typically $1.1^{\prime \prime}$ compared to $0.6^{\prime \prime}$ in the HSC images). However we checked that these objects are very likely stars according to their colours and images. More precisely, about 20, 40, 60 and $80 \%$ of the matched stars at $i \simeq 21,22,23$ and 24 , respectively, are "galaxies" classified in the S82 catalogue. In other words, these results mean that the S82 catalogue of stars or galaxies at $21 \lesssim i \lesssim 24$ suffer from significant misidentification or non-detection. Also note that there are some cases that multiple HSC stars are matched to a single object in S82, as indicated by the magenta histogram. This is a minor fraction, and we do not use these stars in the following analysis. In summary, the use of HSC data is crucial for a construction of secure star catalogue and therefore for our study.

We also use the matched galaxies, as indicated by the red histogram in Figure 3, for a recalibration of our proper motion measurements. Galaxies in the HSC catalogue are identified based on the method described in Section 3.1. We start with each galaxy in HSC and then identify its counterpart in S82. If the matched object is not classified as a galaxy in S82, we choose not to include it in order to ensure a secure galaxy sample. The HSC catalogue gives a high number density of galaxies, about $15-30 \mathrm{arcmin}^{-2}$ varying with sky regions due to variations in the depths and observation conditions. Thus galaxies are much more abundant than stars on the

\footnotetext{
4 There might be a population of stars that move more than the search radius of 1 " between the time difference of the two datasets, which would be good candidates of hyper-velocity stars. This is beyond the scope of this paper, and will be studied in a separate paper.
}

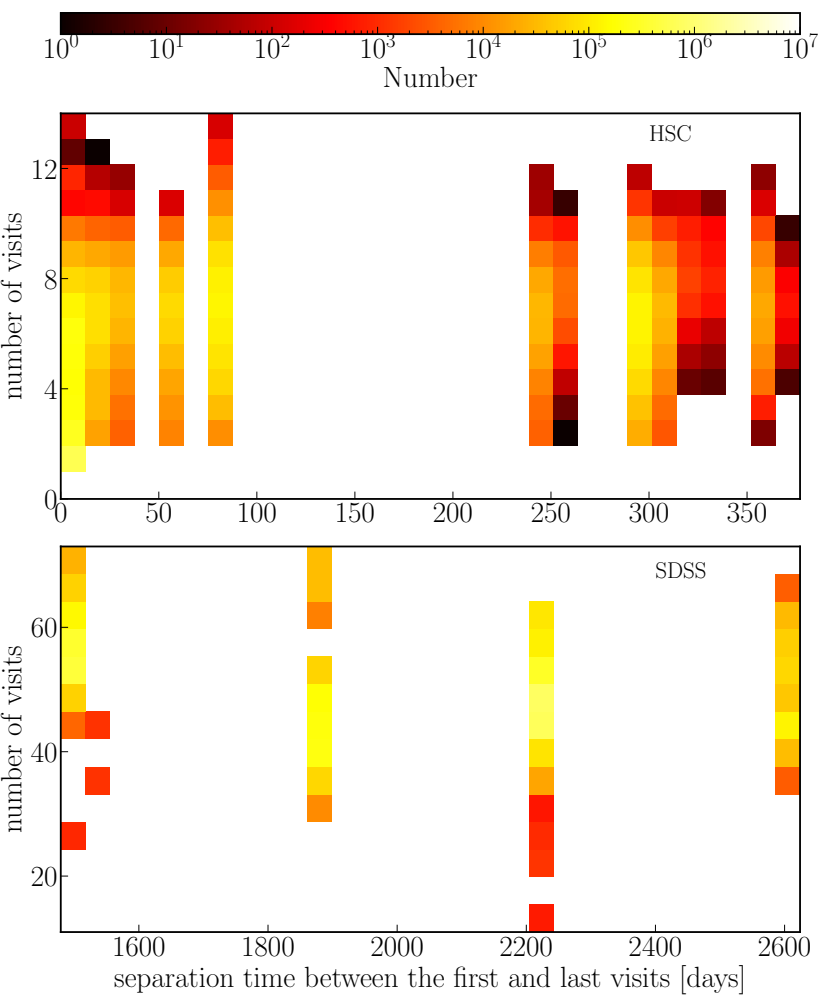

Figure 4. The HSC $i$-band and S82 $r$-band coadd images are constructed from the data taken by multiple visits of each field. The coaddition is done based on each HSC "tract" region or each SDSS CCD "frame", respectively. Upper: Colour in each grid denotes the number of HSC tracts lying in a given value of the 2D space, parametrised by the number of visits of each tract ( $y$-axis) and the separation time between the first and last visits of the tract ( $x$-axis). Lower: The similar result, but for the S82 CCD frames. Based on these information we compute the mean epoch of the coadd image for each matched galaxy/star, and then compute the time baseline for the proper motion measurements.

sky. We have about 3 million matched galaxies. In some cases, we have multiple matches: one object in S82 is matched to multiple galaxies in HSC, as shown by the brown histogram in Figure 3. We do not use the multiply-matched galaxies for the recalibration.

Similarly, we perform a matching of spectroscopicallyconfirmed quasars in the SDSS DR14 catalogue and stars in the Gaia DR2 catalogue to objects in the HSC and S82 catalogues. We have about 9,000 matched quasars and 0.12 million matched stars with Gaia, respectively (see Table 1). We will use the quasars and the Gaia stars for validation and further tests of our measurements.

\subsubsection{An initial estimate of proper motion for each object}

To measure proper motions of matched stars, we first estimate the angular offset of each star between the HSC and S82 catalogues, and then divide the offset by the time difference between the two datasets as an estimate of its proper motion, by making use of the long time baseline, typically 12 years. However, the angular offsets are estimated from the coadd catalogues, which are based on multiple exposures, and we need to be careful about how to estimate the representative epoch of each coadd image. A reasonable choice would be the mean epoch of input single-exposure images in each field. Since we use the $i$-band positions for sources in HSC, we 
use the multiple $i$-band exposures to calculate the mean epoch. For $\mathrm{S} 82$, on the other hand, we use $r$-band individual exposures, though given the drift-scanning mode of observation, the epoch difference calculated from other SDSS bands are almost the same.

In Figure 4, we show the distributions for the number of visits and the time differences between the first and last visits for the HSC and S82 coadd catalogues. The HSC $i$-band coadd images are composed of 2 to 14 visit exposures. The spread of $x$-axis denotes that most of the data are taken within 100 days, i.e. 3 months, and the other data are taken within 360 days, i.e. one year (see Aihara et al. 2018a, for the survey strategy). The $\mathbf{S} 82$ coadd images are built from 11 to 73 visit exposures (mostly 30 to 60 visits), and the separation time has a wide spread. Many data are taken within 4, 5 , 6 and 7 years between 1998 and 2005 .

For each coadd source, we calculate its mean modified Julian date (MJD) based on epochs of all individual exposures from which its coadd image is made, for both HSC and S82. Objects in the same tract of HSC or the same frame of SDSS have the very similar (or same) mean MJD dates. We then adopt the separation time between the mean HSC and S82 modified Julian dates for each object as the time baseline used for the proper motion measurements. The time baseline ranges from 10.1 to 14.6 years for objects in the HSC-S82 region. If we instead fix 12 years time baseline for all objects in the

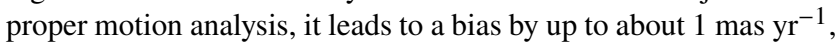
as we will discuss later and in Appendix B.

For each matched star (see Section 3.1), we first estimate its angular offset between the HSC and S82 catalogues, and then divide the angular offset by the time baseline for the star to obtain an "initial" estimate of its proper motion. Note that neither HSC nor SDSS have corrected for the proper motions of stars before image coaddition, and the reference frames for HSC and S82 observations can be different. Although S82 has its own light-motion catalogue (Bramich et al. 2008), for which recalibration of the reference system has been done using galaxies and by taking a mid-epoch run as reference, the star images are in fact not corrected for their proper motions before coaddition, source detection and solving for astrometry. Hence there are likely systematic errors in astrometric solutions in both datasets and also the initial proper motion estimates if we do not correct for the difference in reference frames. In order to correct for the systematic errors, we will use the catalogue of matched galaxies, constructed in Section 3.1, by making use of the fact that galaxies should not move on the sky and can serve to define the reference coordinates. We will describe our recalibration method in the next section.

\subsection{Recalibration}

We use apparent angular offsets of galaxies between the HSC and S82 catalogues to correct for systematic errors in the astrometric solutions of the two datasets. Here we describe details of the recalibration method.

\subsubsection{Astrometry recalibration in the proper motion measurements}

Quasars and galaxies are distant objects that should not have any proper motions. Hence, any apparent angular offsets of galaxy/quasar positions between HSC and S82, if found, reflect systematic errors in the astrometric solutions of the two datasets. Conversely, we use the observed angular offsets of galaxies/quasars to recalibrate the systematic errors (e.g. see Koposov et al. 2013, for a similar method). In order to correctly capture how the systematic errors vary with different regions of the sky, we require a sample of galaxies or quasars to have high enough surface number density to measure the apparent angular offsets at each position on the sky at a high statistical significance. There are only $\sim 9,000$ matched quasars within our footprints (see Table 1), which is too sparse, while there are about 3.2 million matched galaxies, with the mean surface number density of $32,000 \mathrm{deg}^{-2}$ (Table 1). For this reason we use galaxies to perform the astrometry recalibration.

We build a "recalibration" map to correct for the systematics of the angular offset for each matched star at an arbitrary position within the overlapping Stripe 82 region of $100 \mathrm{deg}^{2}$. To do this, we need to well capture possible spatial variations in the systematic effects. The smallest spatial scale would be a size of the CCD chip or frame. The SDSS frame size is about $0.225 \times 0.150 \mathrm{deg}^{2}(2048 \times 1361$ pixels with mean pixel size of about $0.396^{\prime \prime}$ ) (Gunn et al. 1998), while the HSC CCD chip size is about $0.196 \times 0.096 \mathrm{deg}^{2}(4176 \times 2048$ pixels with mean pixel size of about $0.168^{\prime \prime}$ ) (Miyazaki et al. 2018). Hence we split the sky footprint into small grids with size of $0.05 \times 0.05 \mathrm{deg}^{2}$ each, which is chosen to be smaller than the frame or CCD size, and thus is able to capture spatial variations in the systematic errors within one frame or CCD chip, if exists. At the meanwhile, the chosen grid size contains an enough number of galaxies for the recalibration.

We adopt 66,000 grids in total that cover the overlapping region of HSC and S82, including the footprint boundary regions without any matched galaxy inside. We have about 80 galaxies per grid on average, with the number density shown in the upper panel of Figure 5. For each grid, we compute the mean angular offset of matched galaxies between HSC and S82 after $3 \sigma$ clipping based on their angular offset distributions in the grid. Then we divide the angular offset by the mean time separation, estimated based on the method in Section 3.2.2, to convert the angular offset into units of proper motions in R.A. and Dec. directions, i.e. mas $\mathrm{yr}^{-1}$, in each grid $^{5}$. We checked that, even if we exclude galaxies that are near or within the stellar locus in colour-colour space in Figure 2, to minimise a possible contamination of stars to the galaxy sample, the following results are almost unchanged. We also checked that for different grid sizes chosen to be smaller than $0.1 \times 0.1 \mathrm{deg}^{2}$, the following results are almost unchanged.

The middle panel of Figure 5 shows the map of mean angular offsets. We can see spatial structures for the amplitudes of angular offsets. Along the R.A. direction, the structures are smoother and finer, which is the drift-scan direction of SDSS. Along the Dec. direction, there are about 12 distinct, discontinuous structures. This structure reflects the 2 scan directions by the 6 CCD chips of SDSS camera in the $S 82$ region (see Figure 1 in Jiang et al. 2014). Thus the astrometry accuracy in our measurements is limited by the quality of SDSS data, not by the HSC data. The degree-scale structures seem to reflect depth variations of the data, and the finer structures would reflect structures inside the CCD chips. The standard devia-

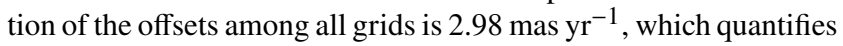
the level of spatial fluctuations in the footprint. In fact, we found that, to measure the tangential velocity dispersions of stars at large

5 The division of the galaxy angular offsets by the time baseline is just for our convenience. Another way of the recalibration is i) first subtract the galaxy angular offset from each star, contained in the corresponding grid of galaxies, and ii) measure the proper motions of stars. This is equivalent to our method, and just the order of computation processes is different, because the time baselines for galaxies and stars in the same frame/CCD chips are the same. 

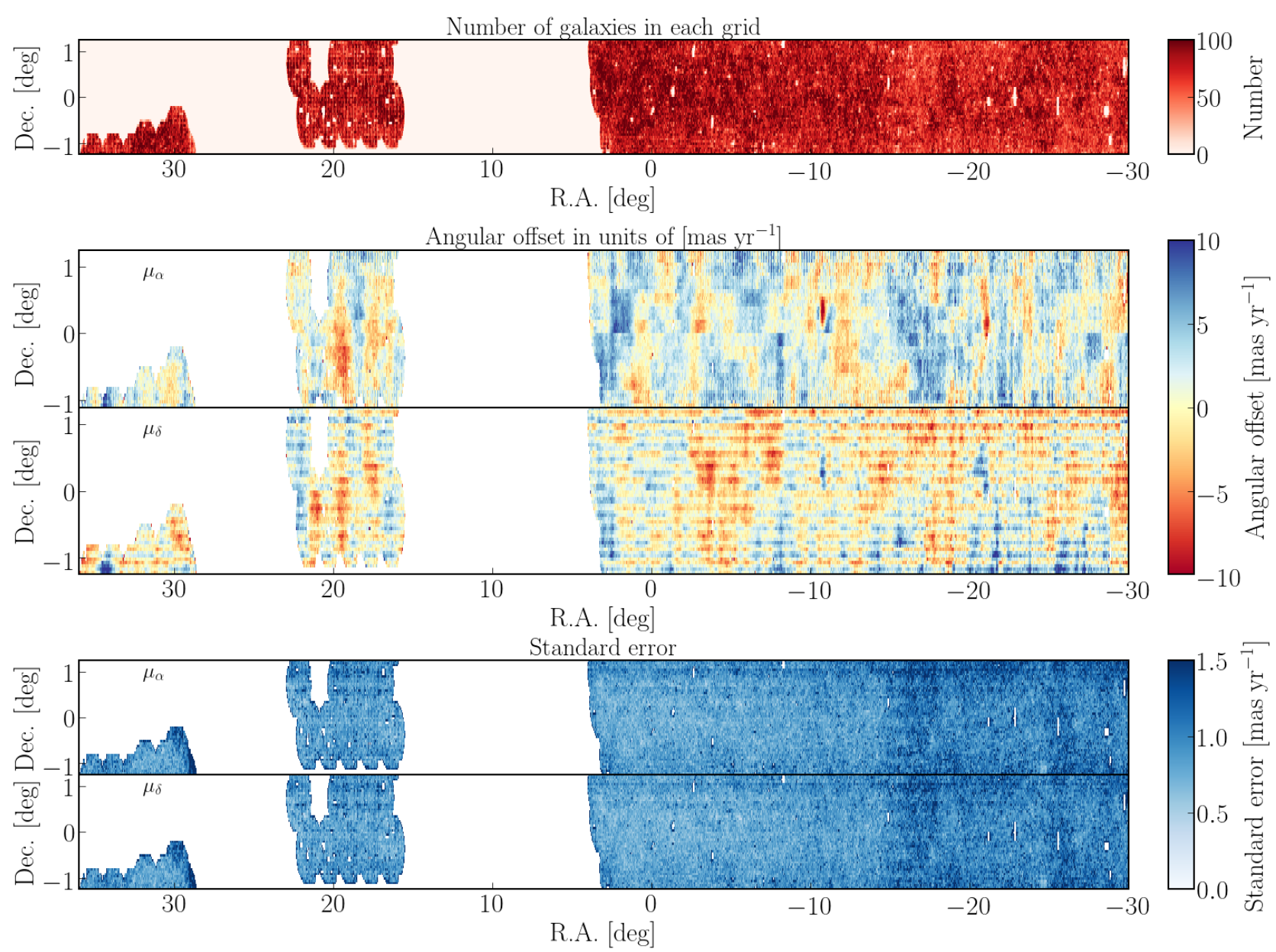

Figure 5. Recalibration map of astrometric solutions between HSC and S82, obtained using the matched galaxy sample (see text for details). The map is given by 66,000 grids in total, each of which has a size of $0.05 \times 0.05 \mathrm{deg}^{2}$. Upper panel: The number of galaxies in each grid, which contains about 80 galaxies on average. Middle: The mean angular offset of galaxies in each grid between HSC and S82, computed by averaging the astrometric R.A. and Dec. positions of

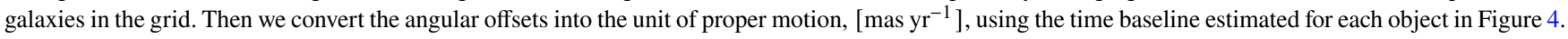
Lower: The 1- $\sigma$ error on the mean angular offset per year in each grid. The error is dominated by the measurement errors of galaxy centroid positions.

distances, it is important to take into account the spatial variations of the recalibration map, as we will discuss later (see Appendix C).

We also estimate the 1- $\sigma$ errors of the mean angular offset in each grid, estimated from the variance of angular offsets of galaxies in the grid, as shown in the lower panel. We typically have a $1 \sigma$ accuracy of 0.96 mas $^{-1}{ }^{-1}$. We checked that the statistical error is mainly attributed to measurement errors of the centroid positions for galaxies in the catalogues. However, it turns out that the statistical errors quoted in the catalogues underestimate the true errors, and we will later discuss a more careful estimation of the statistical errors in the proper motion measurements in Appendix C.

To make a recalibration of the proper motion measurements, we first find the closest grid of galaxy offset map (Figure 5) to each matched star, and then subtract the mean angular offset per year of galaxies from the measured proper motion of the star. This is our fiducial dataset of the proper motion measurements.

\subsubsection{Photometric recalibration}

The HSC griz filter system (transmission curve, wavelength coverage, etc.) is very similar to, but not exactly the same as the SDSS filter system. As will be described in detail in Section 3.4, we adopt the method of Jurić et al. (2008) and Ivezić et al. (2008) to infer photometric distance for each star based on its colour, in which the relation linking the colour to intrinsic luminosities is calibrated based on the photometry system of SDSS. Hence we need to make colour transformation of the HSC photometry of stars to infer the SDSS photometry, i.e., recalibrate the HSC $g, r$ and $i$ photometry against the SDSS photometry.

We first pick up stars in the $68 \%$ densest grids over the colourcolour diagram in Figure 2, which helps to significantly reduce the number of stars with large photometric errors and hence scattered away from the stellar locus of main-sequence stars. We also only focus on stars brighter than $i_{\mathrm{HSC}, \mathrm{PSF}}=23$. For these stars in HSC, we compute their $g, r$ and $i$ PSF magnitude differences from SDSS PSF magnitudes $\left(g_{\mathrm{HSC}, \mathrm{PSF}}-g_{\mathrm{S} 82, \mathrm{PSF}}, r_{\mathrm{HSC}, \mathrm{PSF}}-r_{\mathrm{S} 82, \mathrm{PSF}}\right.$ and $\left.i_{\mathrm{HSC}, \mathrm{PSF}}-i_{\mathrm{S} 82, \mathrm{PSF}}\right)$ against $\mathrm{HSC}\left(g_{\mathrm{PSF}}-r_{\mathrm{PSF}}\right),\left(r_{\mathrm{PSF}}-i_{\mathrm{PSF}}\right)$ and $\left(i_{\mathrm{PSF}}-z_{\mathrm{PSF}}\right)$ colours, respectively. We adopt the second order polynomial models to fit and describe how the HSC and SDSS PSF magnitude difference in each band depends on colour on average, and use the best-fit models to recalibrate the HSC photometry of stars against SDSS. 


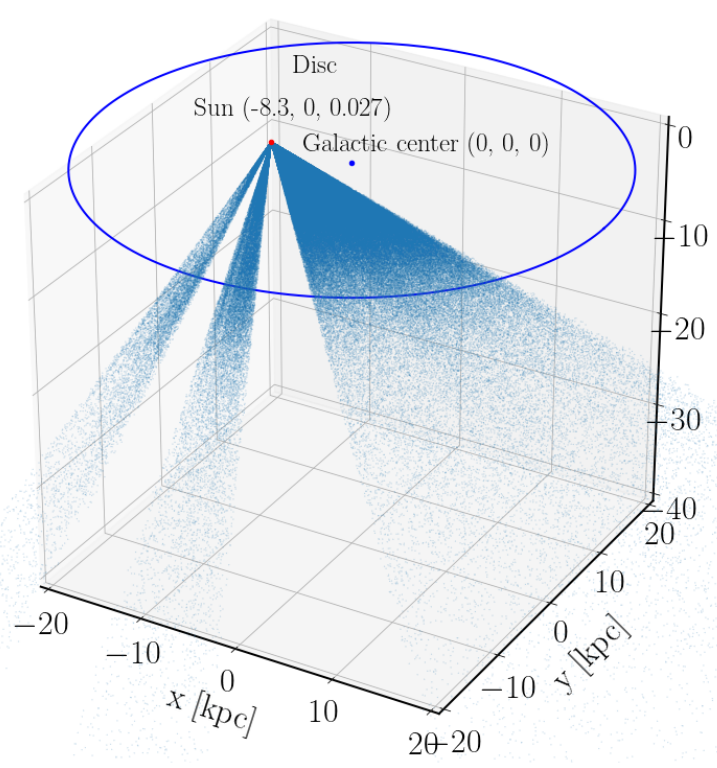

Figure 6. The 3-dimensional distribution of HSC-S82 matched stars (lightblue dots). We derive the 3D positions from the angular position in the Equatorial coordinate (R.A. and Dec.) and photometric distance for each star. Red and dark-blue dots denote the position of the Sun and the Galactic centre. The Galactic disc plane is represented by the blue circle. The positive $x$ and $z$ directions are defined to point towards the Galactic centre and the north Galactic pole.

The recalibration not only affects our determination of photometric distance measurements, but also affects the proper motion measurements after correcting for our own motion (see Section 4.3 for details), because our own motion affects the observed proper motions for stars at different distances by different amount. Moreover, it slightly affects how we divide stars into red and blue populations. However, as we have checked, the average amount of recalibration in HSC $g, r$ and $i$-bands is in fact quite small. For blue stars with $g_{\mathrm{PSF}}-r_{\mathrm{PSF}}<0.6$ (after photometric recalibration), the average amounts of recalibrations in $g, r$ and $i$ PSF magnitudes are $0.0254,0.0039$ and -0.0126 , respectively. For red stars with $g_{\mathrm{PSF}}-r_{\mathrm{PSF}}>0.6$ (after photometric recalibration), the average recalibrations are slightly larger, which are $0.1369,0.0112$ and 0.0859 in $g, r$ and $i$ PSF magnitudes, respectively. We have explicitly checked that, after including the photometric recalibration, the

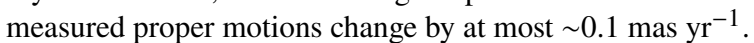

\subsection{Photometric distance}

We will combine the proper motion measurements with the distance information of each star to exploit the three-dimensional structure of the velocity field. We use the method in Jurić et al. (2008) and Ivezić et al. (2008) to estimate the photometric distance of each star. The readers can find more details in the two papers, and in this subsection we briefly introduce our main approach.

As mentioned in Jurić et al. (2008), main-sequence stars have a well-defined colour-luminosity relation. One can thus estimate an intrinsic luminosity of each main-sequence star and hence the distance from the measured colours and fluxes. Following the "photometric parallax" relation provided by Jurić et al. (2008), we esti- mate the photometric distances of main-sequence stars using their $g_{\mathrm{PSF}}-i_{\mathrm{PSF}}$ colours and $r_{\mathrm{PSF}}$ magnitude. Since we always adopt PSF magnitudes in different filters to calculate colours for stars, hereafter we neglect the lower index of "PSF" when quoting the colours. In addition to the colour, accurate photometric distance estimates also depend on metallicities. Ivezić et al. (2008) developed polynomial models which relate effective temperature and metallicity of mainsequence stars to SDSS $u-g$ and $g-r$ colours. The models are based on the effective temperature and metallicity derived from the spectroscopic data of 60,000 F- and G-type main-sequence stars with $g-r$ colours in the range of 0.2 and 0.6 .

In principle, one can estimate the metallicity through $u-g$ and $g-r$ colours following the approach of Ivezić et al. (2008). However, HSC does not have $u$-band data. The SDSS $u$-band filter, unfortunately, is shallow and gives quite large photometric errors for faint stars, and thus the errors of photometric distances are dominated by $u$-band photometric errors. The typical S $82 u$-band PSF magnitude error is as large as 1-1.5 mag at HSC $i$-band magnitudes of $21<i_{\mathrm{HSC}, \mathrm{PSF}}<24.5$, and across this magnitude range, SDSS basically measures the sky flux rather than true source detections. The error drops below 1 mag at brighter magnitudes, but is still quite large. The typical errors are $0.6,0.3$ and $0.1 \mathrm{mag}$ at $i_{\mathrm{HSC}, \mathrm{PSF}} \sim 20$, 19 and 18, respectively. For comparison, the median photometry errors of our HSC-S82 matched stars in HSC $g, r$ and $i$-band are only about $0.0133,0.0065$ and $0.0033 \mathrm{mag}$, respectively; these errors are significantly smaller.

As have been pointed out by Jurić et al. (2008), a 0.1 mag of error in colour can lead to $0.5 \mathrm{dex}$ of error in the metallicity estimate, which corresponds to a relative distance error up to $20 \%$. To ensure accurate photometric distance estimates, the S82 $u$-band data is almost useless beyond $i_{\mathrm{HSC}, \mathrm{PSF}} \sim 18$. Hence it is difficult to achieve proper metallicity estimates based on the colour for stars fainter than $i_{\mathrm{HSC}, \mathrm{PSF}} \sim 18$.

Ivezić et al. (2008) investigated the $[\mathrm{Fe} / \mathrm{H}]$ distribution of disc and halo stars out to $\sim 10 \mathrm{kpc}$. In their results, halo stars with $|z|>4 \mathrm{kpc}$ show a quite homogeneous spatial distribution of $[\mathrm{Fe} / \mathrm{H}]$, and they are dominated by blue stars. The value of $[\mathrm{Fe} / \mathrm{H}]$ peaks at -1.45 and follows a nearly Gaussian distribution with 1- $\sigma$ scatter of about 0.41 dex (see Figure 10 in Ivezić et al. 2008). On the other hand, nearby disc stars are mostly red stars. It is more difficult to properly determine the metallicity for red stars, but the mean $[\mathrm{Fe} / \mathrm{H}]$ of disc stars is about -0.6 (Ivezić et al. 2008).

Thus throughout this paper, we adopt a fixed metallicity of $[\mathrm{Fe} / \mathrm{H}]=-1.45$ to estimate the photometric distances for all stars (both red and blue stars) ${ }^{6}$, if the initial distance estimate is greater than $6.25 \mathrm{kpc}$; that is, we ignore the metallicity dependence for such halo stars. This mainly picks up stars with $|z|>4 \mathrm{kpc}$, i.e., stars away from the Galactic disc. For more nearby blue stars, whose photometric colours are more accurately measured, we estimate their metallicities based on the $u-g$ and $g-r$ colours, with the $u$-band flux taken from S82 and $g, r$-band fluxes taken from HSC after photometric recalibrations against SDSS (see Section 3.3.2), though the total number of nearby blue stars is very small. For nearby red stars at $<6.25 \mathrm{kpc}$, we keep their metallicities fixed to $[\mathrm{Fe} / \mathrm{H}]=-0.6$ following Ivezić et al. (2008).

Figure 6 shows the 3-dimensional spatial distribution for our sample of matched stars, based on their Equatorial coordinates and

\footnotetext{
6 We also tried to assign each blue star with a value of $[\mathrm{Fe} / \mathrm{H}]$ from the Gaussian distribution with $\langle[\mathrm{Fe} / \mathrm{H}]\rangle=-1.45$ and $\sigma=0.41 \mathrm{dex}$, and our results change very little.
} 
photometric distances. The three disjoint HSC-S82 footprints all point towards negative $z$ directions with respect to the Galactic disc plane. Thus, except for those very nearby stars, which might include some thick disc stars, the majority of matched stars are in the halo region. The HSC-S82 regions are distributed in the range of the Galactic latitude, $b \simeq[-64,-40] \mathrm{deg}$.

However, halo stars could have lower metallicities, e.g. $[\mathrm{Fe} / \mathrm{H}] \sim$ -2 , than our default value of -1.45 , as indicated in An et al. (2013). In Section 5 we will discuss how possible uncertainties in the metallicities affect the photometric distances and in turn affect our proper motion measurements for halo stars.

\subsection{Correction for differential chromatic refraction}

After a careful analysis, it turned out that the differential chromatic refraction effect (hereafter DCR; Filippenko 1982) by the Earth's atmosphere gives a subtle systematic effect in our proper motion measurements. We need to correct for the DCR effect to achieve

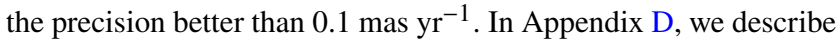
details of our correction method of the DCR effect, and also give a validation of the method using the SDSS DR14 spectroscopic quasar sample.

The HSC data are not affected by DCR, because the HSC widefield corrector is equipped with the DCR corrector, more exactly the Atmospheric Dispersion Compensator (ADC) as described in Miyazaki et al. (2018). On the other hand, the SDSS camera does not have such a DCR corrector. DCR stretches an observed image of source along the zenith direction due to the wavelength-dependent refraction by atmosphere and, as a result, each object appears to be observed at a shifted position in the camera image by a different amount in different filters. For SDSS, because of its specific driftscanning mode of observation, the zenith direction is mainly along the Dec. direction for the Stripe 82 region. Since the spectral energy distribution (SED) of galaxies and stars are different, our astrometry recalibration method using the galaxy positions (Figure 5) cannot fully correct for the DCR effects for stars.

We correct for the DCR effect by using the Gaia DR2 proper motions as a reference sample. Because the space-based Gaia data are not affected by DCR, the difference between our measured proper motions and Gaia data reflects the amount of DCR effect. To do this, we first cross match our sample of stars with the Gaia DR2 catalogue based on their positions. Since Gaia stars are brighter than $G \sim 21$, we can only match bright and nearby stars; the matching gives 117,703 stars in total in the HSC-S82 region, as given in Table 1. As for our default method of correcting for the DCR effect, we first measure the DCR effect as a function of the $g-i$ colours for the matched stars, averaged over the survey footprints. When we measure a proper motion for each star, including other stars (nonGaia matched stars), we use the Gaia measurement to correct for the DCR effect based on the $g-i$ colour of the star, assuming that the DCR effect is characterised by the colour, and does not depend on the distance of the star. The details are presented in Appendix D.

\section{RESULTS}

Following the methods described up to the preceding section, we estimate proper motions for about 0.55 million stars down to $i \simeq 24.5$. In this section we show the main results of this paper. We first give a validation of our proper motion measurements by comparing with the Gaia results in Section 4.1. Hence readers who are interested in the results can directly go to Section 4.2.

\subsection{Validation of our method: comparison with Gaia}

Before going to the main results, we first demonstrate a validation of our method from the comparison with the Gaia measurements of proper motions that have an exquisite precision (Gaia Collaboration et al. 2018). To do this, we first cross-match our sample of stars with the Gaia DR2 catalogue based on their positions. Since Gaia stars are brighter than $G \sim 21$, we can only match bright and nearby stars; the matching gives 117,703 stars in total in the HSC-S82 region, as given in Table 1.

In Figure 7, we show the proper motion measurements for the matched stars, which are estimated by averaging the proper motions of stars that reside in each bin of R.A. ( $x$-axis). Throughout this paper, for plots showing the averaged proper motion measurements, we use the median in a given bin to denote the central value of the measurement, and show the asymmetric errorbars to denote statistical errors in the median, computed by

$$
\begin{aligned}
\sigma_{+} & \equiv \sqrt{\frac{\pi}{2}} \times \frac{1.428\left(q_{75}-q_{50}\right)}{\sqrt{N}}, \\
\sigma_{-} & \equiv \sqrt{\frac{\pi}{2}} \times \frac{1.428\left(q_{50}-q_{25}\right)}{\sqrt{N}}
\end{aligned}
$$

for the upper- and lower-side errorbars, respectively, where $q_{25}, q_{50}$, and $q_{75}$ are the 25, 50, and 75 percentiles, respectively, and $N$ is the number of stars (or galaxies) in the bin (Ivezić et al. 2014).

The top and middle panels of Figure 7 clearly show that our method gives a significant detection of the proper motions and our results well reproduce the Gaia results for both components of $\mu_{\alpha}$ and $\mu_{\delta}$. The lower panel quantifies the agreement. The averaged difference between the proper motions of HSC-S82 and Gaia for all the matched stars is remarkably close to zero, more precisely only about $\mu_{\alpha} \simeq-0.0007 \mathrm{mas}^{-1}$ and $\mu_{\delta} \simeq-0.0010 \mathrm{mas} \mathrm{yr}^{-1}$. We note that the good agreement is a result of the DCR correction, which is by design as we used Gaia as a reference (see Section 3.5 for details). A similar plot before correcting for the DCR effect is provided in Appendix D, which still shows farily good agreement. More precisely, the averaged difference between the proper motions of HSC-S82 and Gaia is $\sim 0.05$ mas yr$^{-1}$ along Dec., which is about 50 times larger than in Figure 7. In addition, the validation test using quasars shown in Section D2 is independent and convincing as well. A closer look at Figure 7 reveals that the difference from Gaia is relatively larger at R.A. $\sim 20$ deg., where the region contains more blue stars from the Sagittarius stream. This is because the DCR effect is expected to be stronger for bluer objects in our sample. We also show in Appendix D that we see a more significant difference in the Gaia comparison for blue stars.

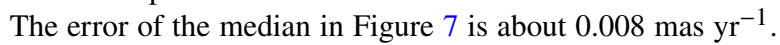

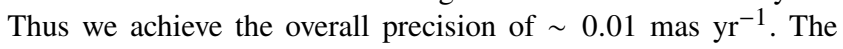
figure also shows that the error is much smaller than the width of the difference distribution $\left(\sim 2.2\right.$ mas $\left.\mathrm{yr}^{-1}\right)$ that mainly reflects the statistical errors of proper motions for individual stars (see below). Thus these results demonstrate the power of the statistical method for the accurate proper motion measurements.

Most of the matched stars have $20<G<21$ in the Gaia band, which is near the flux limit of Gaia sample. The median measurement errors for individual stars in the Gaia data are typically $\left(\sigma_{\mu_{\alpha}}, \sigma_{\mu_{\delta}}\right)=(1.37,1.10)$ mas $\mathrm{yr}^{-1}$. Compared to this, the measurement errors for individual stars in the range $20<G<21$

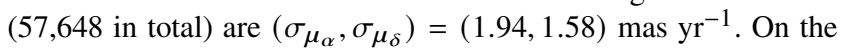
other hand, the measurement errors for the HSC-S82 proper mo-

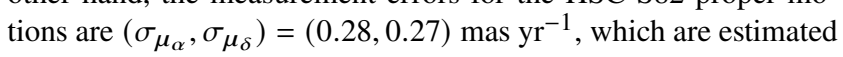
by propagating the measurement errors of the centroids of stars for 

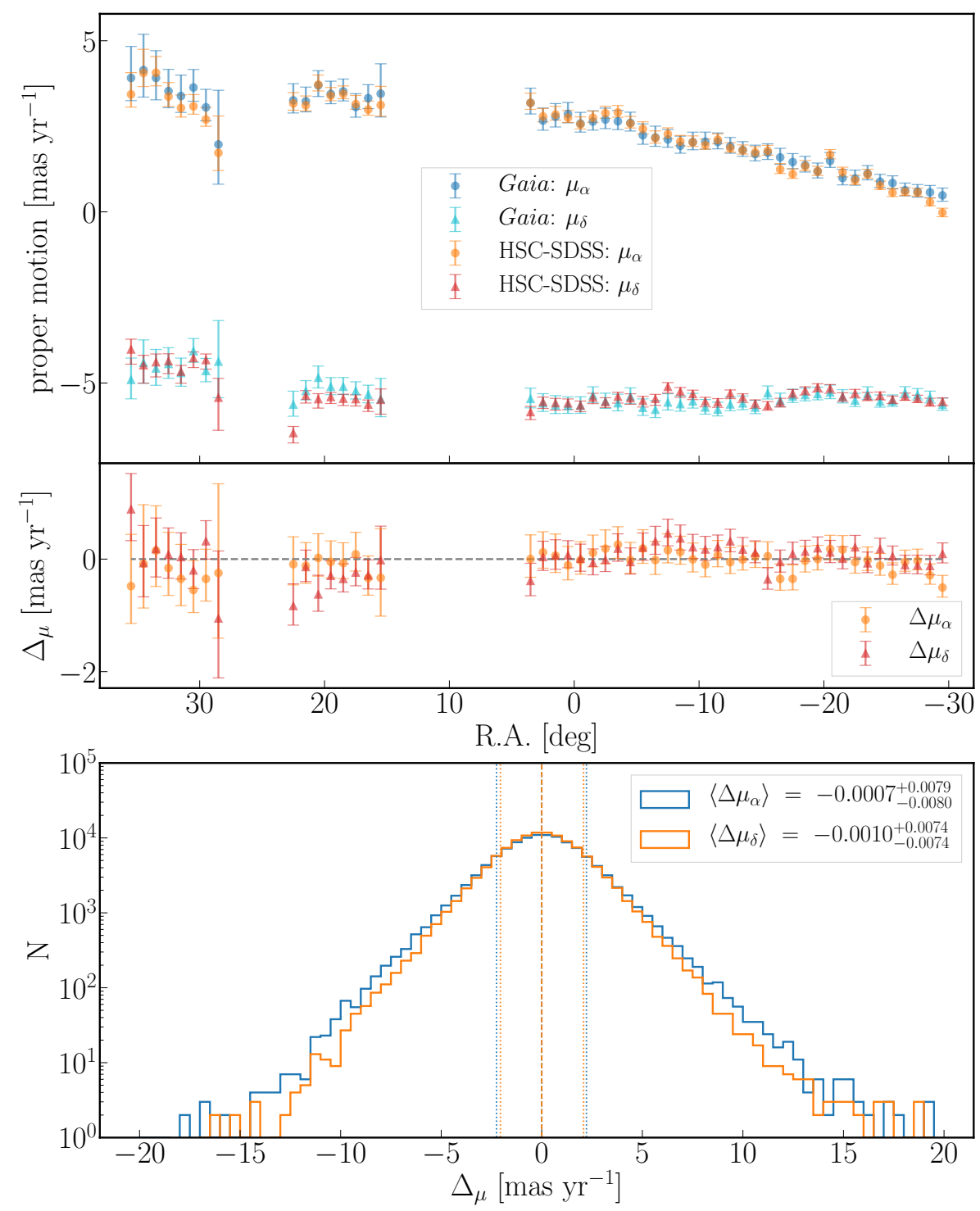

Figure 7. Comparison of our measured proper motions with the Gaia proper motions for the matched stars, where we have about 120,000 matched, bright stars in the overlapping HSC-S82 footprints (see Table 1). The upper panel shows the proper motion measurements, $\left(\mu_{\alpha}\right.$ and $\left.\mu_{\delta}\right)$, obtained by taking the median of proper motions for stars in each bin of R.A. region. Our HSC-S82 proper motions are in good agreement with the Gaia results. The middle panel shows the difference between each component of proper motions in R.A. bins. The asymmetric errorbars show the 1- $\sigma$ errors on the median, estimated using Equation (1). The lower panel shows the distributions of the differences between the proper motion components of HSC-S82 and Gaia for each star in all R.A.

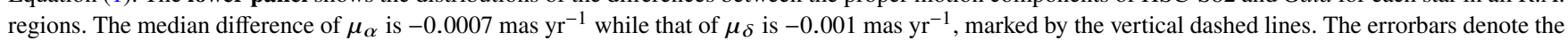
errors of the median computed using Equation (1). The other two vertical dotted lines mark the $68 \%$ width of the whole distribution, which is much wider than the error of the median, reflecting that errors in the proper motion measurements are dominated by the centroid errors of individual stars.

both the HSC and S82 data. Note that the measurement errors in HSC are one order of magnitude smaller than those in S82, meaning that an accuracy of our proper motion measurements is limited by the errors of S82 data. The reader can find more detailed information about the measurement errors in Figure $\mathrm{C} 1$. Thus the statistical error or the width of the proper motion difference distribution in Figure 7 is dominated by the measurement errors of Gaia, not those of HSC-S82 data.

We should note that the agreement in Figure 7 cannot be realised unless we implement the proper HSC-S82 time baseline (see Section 3.2.2) and the astrometry recalibration using the angular offsets of galaxies in Section 3.3. Without the proper time baseline and the astrometry recalibration, our measurement displays a

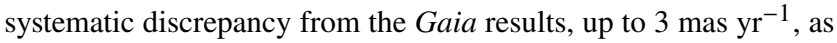
explicitly shown in Figure B1. The astrometry recalibration based on galaxies gives a more significant correction (about 60\% compared to the signal), but both the two effects are important to include. We should also note that the about 12-year time baseline, between HSC and S82, is critical to achieve the precise measurement. The angular offsets of stars between HSC and S82, which are our direct observables, are a factor of 12 greater than the proper motions shown in Figure 7.

We also comment on a possible effect of parallax on the proper motion measurement. Gaia DR2 jointly modelled parallax and proper motion during the continuous 22 months observation. In our HSC-S82 proper motion measurement we do not consider the 


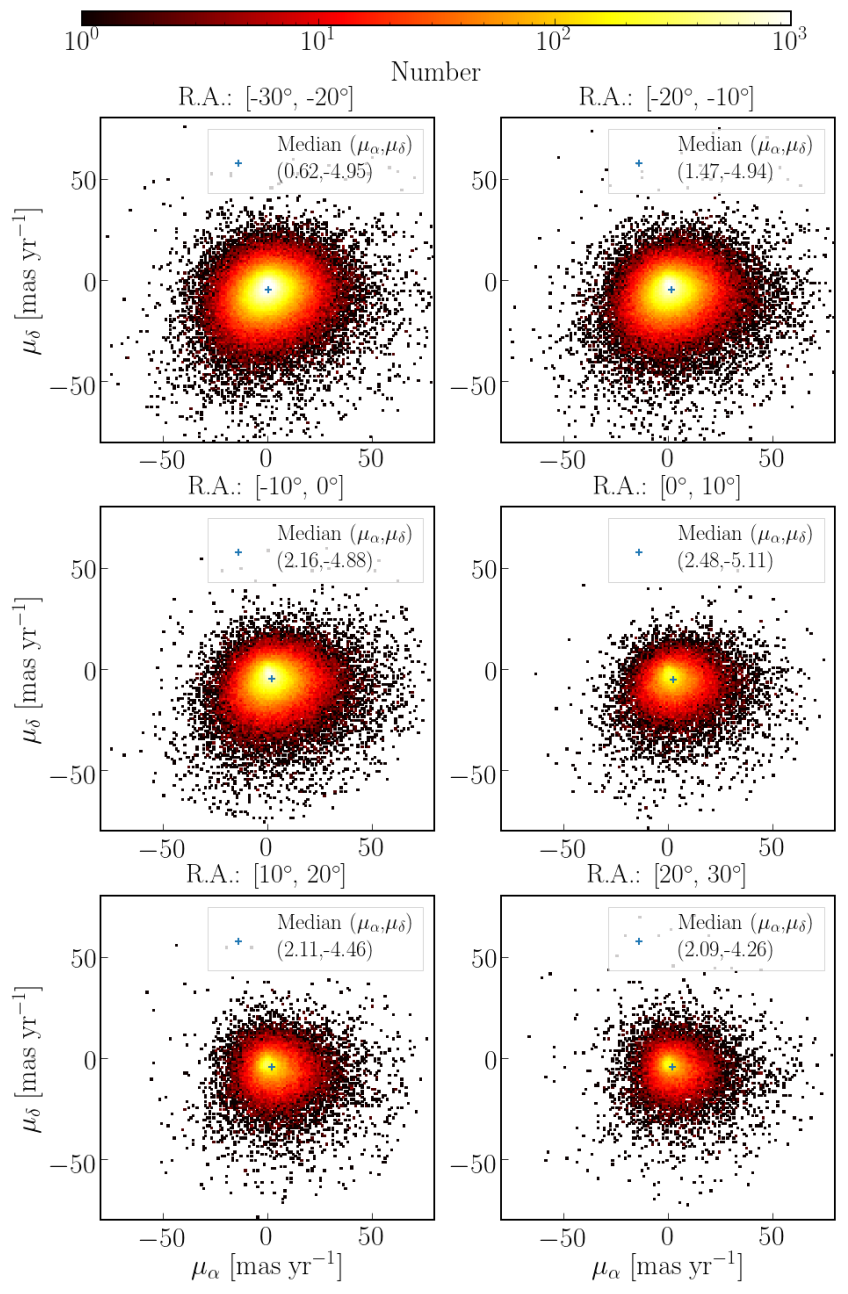

Figure 8. Distribution of stars in the plane of proper motion components, $\mu_{\alpha}$ versus $\mu_{\delta}$. Colour in each grid denotes the number of stars as indicated by the colour bar. Different panels correspond to the results for the different regions divided by the R.A. range. The numbers in the parenthesis in each panel denote the median values of $\mu_{\alpha}$ and $\mu_{\delta}$.

effect of parallax. The parallax amounts to about 1 or 0.1 mas for a star at distance 1 or $10 \mathrm{kpc}$, respectively, and this corresponds to

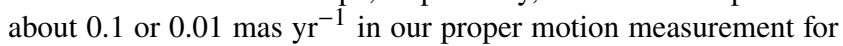
a 10-year time baseline. This effect is safely negligible unless we work on very nearby stars, which is not the case for our study.

\subsection{Direct proper motion measurements}

In Figure 8, we show the distribution of proper motions ${ }^{7}\left(\mu_{\alpha}, \mu_{\delta}\right)$ for subsamples of stars divided based on different R.A. range. Note that we use all the matched stars down to $i \simeq 24.5$ for these results. Each panel displays an anisotropic distribution of stars. While the distribution shows a R.A. dependence, all panels display a coherent proper motion towards the lower right corner; the median of the

7 Generally, $\mu_{\alpha}$ should be $\mu_{\alpha} \cos (\delta)$. Since our region is $-1.25^{\circ}<\delta<$ $1.25^{\circ}$, ignoring $\cos (\delta)$ only leads to less than $0.03 \%$ of difference, and thus we neglect it.

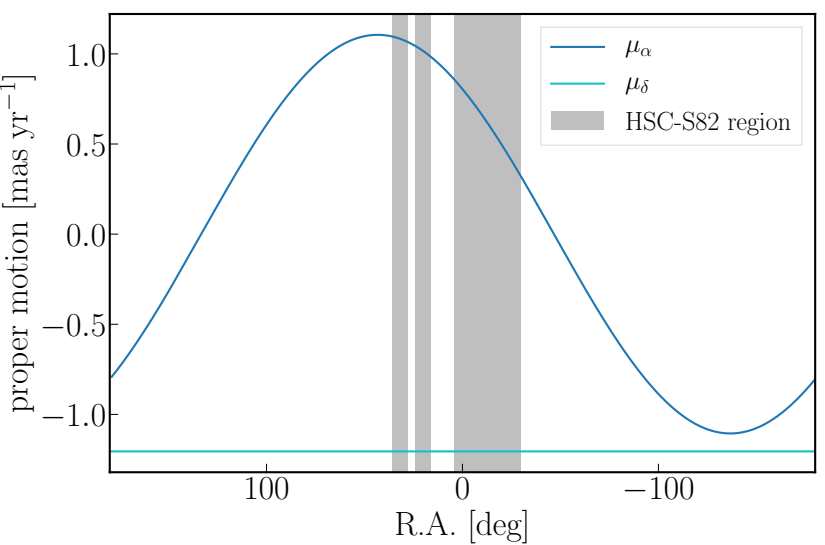

Figure 9. The apparent proper motion of stars due to our own motion if the stars are at rest with respect to the Galactic centre. Here we consider stars at $30 \mathrm{kpc}$ in distance, and we fix Dec. to be zero. We assume that our own motion is a sum of the local standard of rest (LSR) motion with respect to the Galactic centre plus the solar motion with respect to the LSR; we employ $v_{c}\left(R_{\odot}\right)=220 \mathrm{~km} / \mathrm{s}$ for the rotation velocity, and $\left(U_{\odot}, V_{\odot}, W_{\odot}\right)=$ $(11.1,12.24,7.25) \mathrm{km} / \mathrm{s}$ for the motion of the Sun with respect to LSR. Note the apparent motion scales with $1 / d$ ( $d$ is a distance to each star). The shaded regions denote the overlapping HSC-S82 footprints we study in this paper, where the apparent motions in R.A. and Dec. directions are positive and negative on average, respectively. $\mu_{\delta}$ looks like a R.A. independent constant value. This is because the Galactic rotation mainly contributes to $\mu_{\alpha}$ near the Celestial Equatorial region, and $\mu_{\delta}$ is mainly affected by the solar motion with respect to the LSR, which is an order of magnitude smaller.

distribution ${ }^{8}$ indicates a coherent motion with positive value of $\mu_{\alpha}$, and negative value of $\mu_{\delta}$, respectively. We have explicitly checked that the 1- $\sigma$ errors on the median values are much smaller than the absolute median values. Thus our detection here is unlikely caused by the measurement errors.

The trend is consistent with the effect of our own motion ${ }^{9}$ in terms of both its sign and magnitude. The Sun is rotating around the Galactic centre in a counter-rotating manner with the velocity of $\mathbf{v}_{C}\left(R_{0}\right)$. This induces apparent motions of stars with respect to us. For nearby disc stars following the Galactic rotation, the observed proper motions are mainly due to the solar motion with respect to the Local Standard of Rest (LSR), denoted as $\left(U_{\odot}, V_{\odot}\right.$ and $\left.W_{\odot}\right)$. The magnitude is small ( $10 \mathrm{~km} / \mathrm{s})$, but could induce significant proper motions for very nearby stars. In the HSC-S82 footprints, we have explicitly checked that the observed proper motions induced by the solar motion correspond to a motion with positive R.A. and negative Dec. directions, though there are not many disc stars in our sample. For halo stars whose velocities are close to random, the observed proper motions arise from a sum of their true motions with respect to the Galactic centre and the apparent motions due to our own motion. As explicitly demonstrated in Figure 9, the effect

8 The mean shift is very similar to the median after 3- $\sigma$ clipping.

9 We define our own motion to be the motion of our Sun with respect to the Galactic centre, which is a combination of the velocity of the Local Standard of Rest (LSR) and the solar motion with respect to the LSR in the same direction, i.e., $\mathbf{v}_{\odot}=\left(U_{\odot}, v_{c}\left(R_{0}\right)+V_{\odot}, W_{\odot}\right) . v_{c}\left(R_{0}\right)$ is the rotation velocity of the LSR at the solar radius, $R_{0} . V_{\odot}$ is the solar motion with respect to the LSR in the direction of Galactic rotation. $U_{\odot}$ is the velocity towards Galactic centre and $W_{\odot}$ is the velocity component of the solar motion perpendicular to the Galactic disc. 

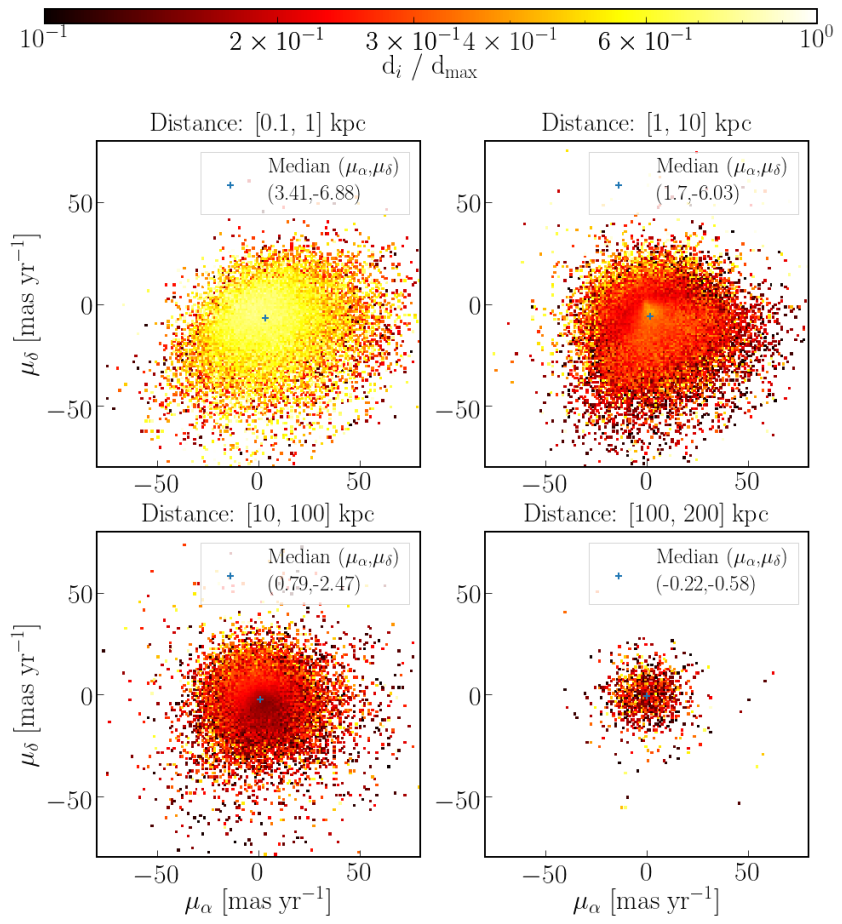

Figure 10. Similar to Figure 8, but each panel shows the distribution of stars divided in the different range of their photometric distances. Colour in each panel denotes the median of photometric distance of stars in each grid, relative to the maximum distance assumed in each plot, $d_{\max }=1,10,100$ or $200 \mathrm{kpc}$, respectively. In each panel, more distant stars tend to display smaller proper motions. Comparing the different panels manifests that the median proper motions and the overall scatters both decrease with the increase in distances.

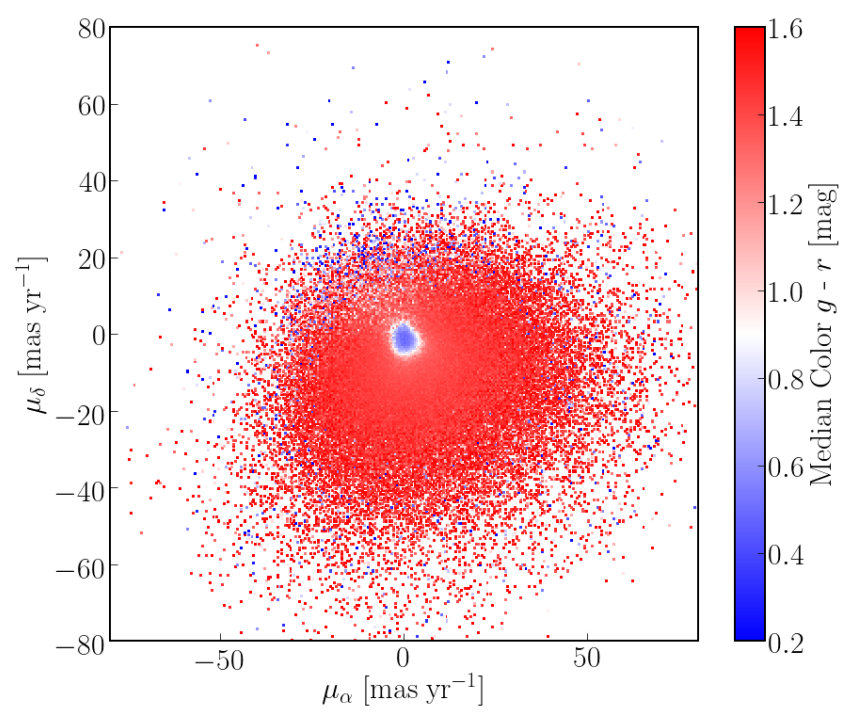

Figure 11. Similar to the previous figure, but the plot shows the distribution for stars divided by their colours, $g-r$. Colour in each grid shows the median of $g-r$ for stars in the grid. Blue stars, which are mainly halo stars, show smaller amplitudes of proper motions, while red stars are more likely nearby disc stars with larger proper motions.
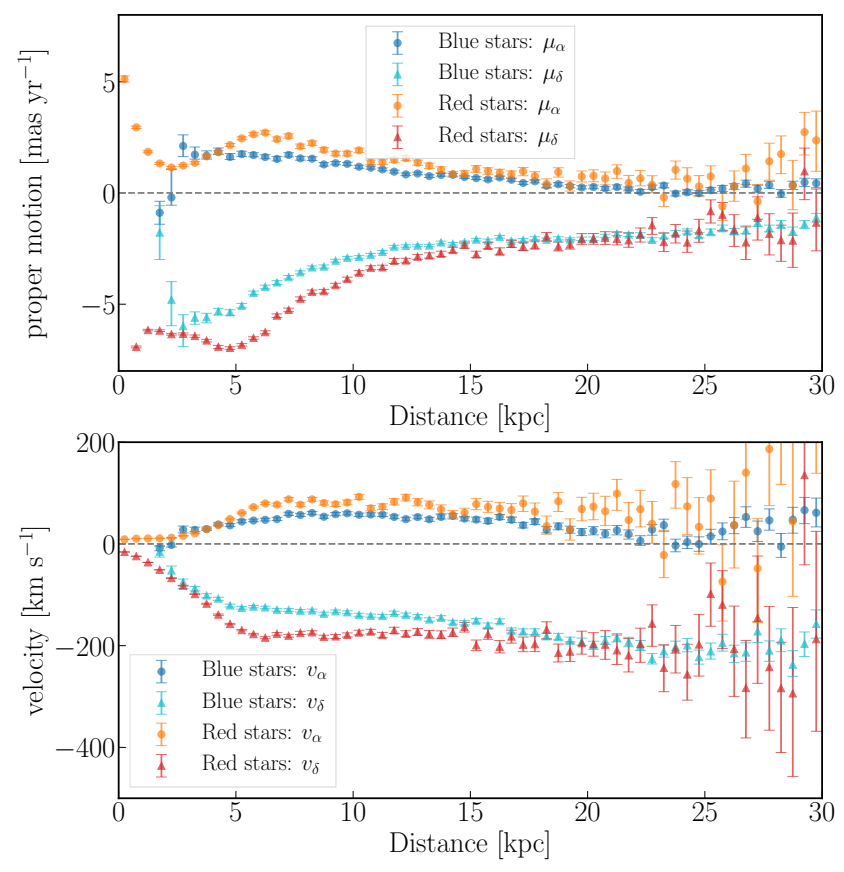

Figure 12. Upper panel: The proper motions of $\left(\mu_{\alpha}, \mu_{\delta}\right)$ measured from the red and blue stars divided into different distance bins out to $30 \mathrm{kpc}$. Here we take the median of proper motions for stars in each bin, after the 3- $\sigma$ clipping of the distribution in each bin. The red and blue stars are divided based on the colour cut of $g-r=0.6$. The errorbars show the $1-\sigma$ errors on the median in each bin, computed by Equation (1). The proper motions shown are direct observables of our data, and the apparent motions due to our own motion are not corrected for yet. Lower: The tangential velocities, which are obtained by multiplying the proper motions by the photometric distances for each star.

of our own motion on more distant stars in the HSC-S82 footprints roughly corresponds to a positive direction in R.A. and a negative direction in Dec., but the exact amount of motions projected onto the Equatorial coordinates is R.A. dependent.

The apparent motion of stars induced by our own motion is expected to show strong dependence on distances of stars, with nearby stars having larger apparent proper motions. We investigate this in Figure 10, which shows the dependence of measured proper motions on photometric distances. The two upper panels of Figure 10 show larger median values and more anisotropic distributions, whereas distant stars in the lower panels display a more isotropic distribution, with smaller median values. In each panel, more distant stars with lighter colours also show larger proper motions. This is consistent with the expectation.

Main-sequence stars with bluer colours tend to be intrinsically brighter, more metal poor and have higher effective temperature, while stars with redder colours tend to be intrinsically fainter. In addition, stars with different metallicities differ in their distances. Those with higher metallicity are younger stars newly formed in the Galactic disc, while the metal-poor stars are old ones, most of which are already located in the halo. We represent the metallicity with the $g-r$ colour. Thus we use intrinsically brighter blue stars to explore the proper motions at large distances. On the other hand, red stars are relatively fainter, and have a larger chance to lie in the Galactic disc at short distances $\lesssim 5 \mathrm{kpc}$. Thus, the colour of stars correlates with the distance. As shown in Figure 11, bluer distant 

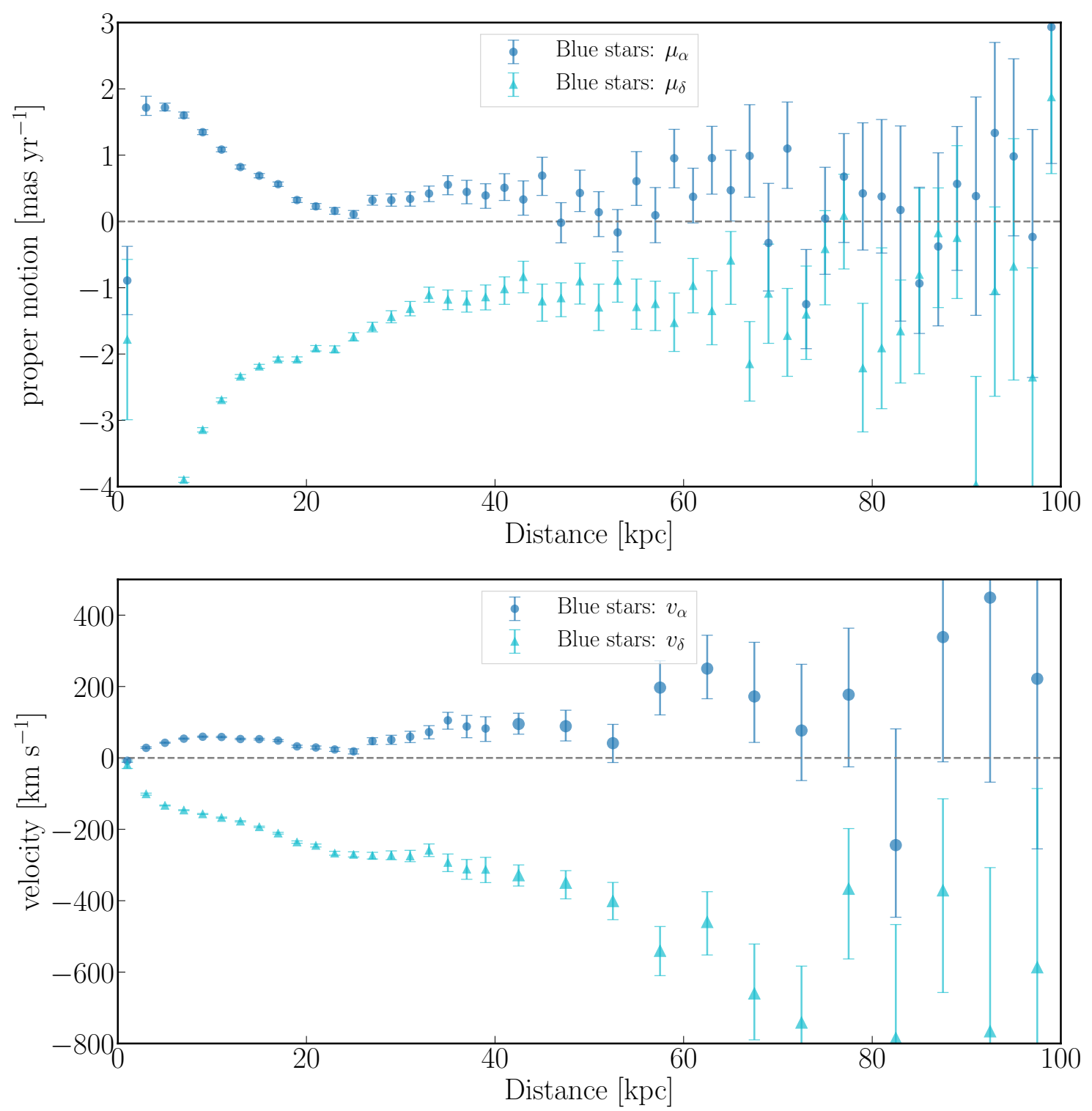

Figure 13. Similar to the previous figure, but the plot shows the net proper motions for blue stars out to $100 \mathrm{kpc}$ in Heliocentric distances. This is our direct observable, which shows a significant detection of the proper motions up to $100 \mathrm{kpc}$. For illustrative purpose, we choose four times wider bin size at $d>40 \mathrm{kpc}$ in the lower panel than those in the upper panel.

stars tend to have smaller proper motions. Interestingly, except for the blue spot in the centre of the plot, there are blue dots scattered in outskirts, indicating the possible existence of distant blue stars moving with high velocities or the existence of some nearby blue stars. In the following, we describe the proper motion results for blue/red stars separately, using the division of $g-r=0.6$.

In Figure 12, we demonstrate how the proper motions and velocities vary with the Heliocentric distances, for red and blue stars. Here we show the results only up to $30 \mathrm{kpc}$ because there are few red stars beyond this range. We first measure photometric distances for individual stars, and then measure the median proper motions for stars divided into different distance bins. The velocities are estimated by multiplying the proper motions by the photometric distances. Thanks to the large sample statistics, we are able to achieve proper motion measurements with a high significance at each distance bin. Within $15 \mathrm{kpc}$, red stars dominate, whereas there are more blue stars beyond $15 \mathrm{kpc}$. Since our own motion has not been corrected here, and those local stars are rotating along with us, this leads to nearly zero velocity at very nearby distances (i.e. only small peculiar velocities with respect to us). The velocities increase to $\geq 200 \mathrm{~km} / \mathrm{s}$ for distant stars beyond $5 \mathrm{kpc}$. As can be seen from Figure 6 , those stars are fairly far away from the disc, whose motions are closer to random. The $\geq 200 \mathrm{~km} / \mathrm{s}$ velocity is mainly due to our own rotation motion in the Galactic disc with respect to the Galactic centre.

More interestingly, in Figure 13, we present the results for blue stars out to $100 \mathrm{kpc}$, where we employ a wider bin width of distances for more distant stars to increase the statistics. For more distant stars, the proper motions decrease, but the figure displays a coherent motion of about $200 \mathrm{~km} / \mathrm{s}$, most of which is caused by our own rotation motion as described below. 

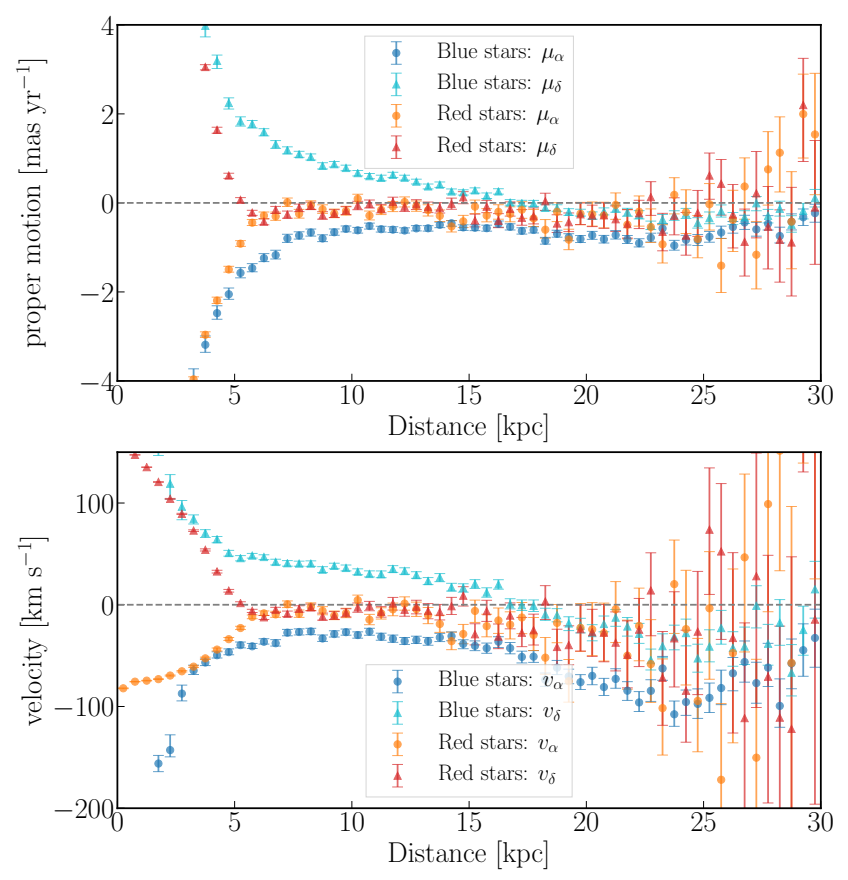

Figure 14. Similar to Figure 12, but the figure shows the the component proper motions and tangential velocities after correcting for the apparent motions due to our own motions for each star.

\subsection{Measurements after correcting for our own motion}

To obtain a physical insight on the proper motion measurements presented in the previous subsection, we need to take into account the effect of our own motion. We use the astropy python package to correct for the effect of solar motion via the following steps:

(i) Input R.A., Dec., proper motion in R.A., proper motion in Dec., line-of-sight velocity and photometric distance to generate an astropy. SkyCoord object.

(ii) Transfer it to the Galactocentric coordinate system.

(iii) Obtain the 3-dimensional positions and velocities with respect to the Galactic centre.

(iv) Subtract our own motion (total motion of the LSR motion with respect to the Galactic centre plus the solar motion with respect to the LSR) from the measured proper motions of stars in 3-dimensional Cartesian coordinates. Here we employ $\left(U_{\odot}, V_{\odot}, W_{\odot}\right)=(11.1,12.24,7.25) \mathrm{km} / \mathrm{s}$ for the Galactic (righthanded) velocity components according to Schönrich et al. (2010), and $v_{c}\left(R_{\odot}\right)=220 \mathrm{~km} / \mathrm{s}$ for the disc rotation.

(v) Transfer back the velocities to those in the International Celestial Reference System (ICRS).

By applying the above steps to each star, we obtain its two components of tangential velocity in ICRS. Note without line-ofsight velocities, we are not able to transfer the tangential velocities to be with respect to the Galactic centre, and thus tangential velocities measured throughout the paper are centred on observers.

In Figures 14 and 15, we show the median proper motions and velocities after subtracting our own motion up to 30 and $100 \mathrm{kpc}$, respectively. These results can be compared with the results in Section 4.2. We have a clear detection of the coherent proper motions up to $\sim 30-40 \mathrm{kpc}$, even after the effect of our own motion is corrected for.
Within $1 \mathrm{kpc}$, there are almost no blue stars. The total tangential velocities of these very nearby red stars are dominated by the Galactic rotation in the thick disc region. Between 1 and $5 \mathrm{kpc}$, the motions of red and blue stars show quite different behaviours. Blue stars show larger tangential velocities, and with the increase in distance, the tangential velocities of both red and blue stars drop, due to the gradually decreased contribution by the Galactic rotation.

Between 5 and $30 \mathrm{kpc}$ of Figure 14, the kinematics of red and blue stars look different. Red stars show very close to zero tangential velocities, while blue stars show non-zero tangential velocities. Stars beyond $5 \mathrm{kpc}$ are expected to be mostly halo stars dominated by random motions, because our HSC-S82 footprints all point towards negative $z$-directions (see Figure 6 ). The none-zero median velocities of blue stars indicate there are some substructures in phase space which contribute to the coherent motion. As we discuss below, the non-zero motion is mainly due to the Sagittarius (Sgr) stream. A part of the Sgr stream is covered within our footprint. The Sgr stream wraps around the Galaxy in an almost polar orbit which is partly overlapped with our observed region. Including the stream in our analysis can significantly affect the median motion of all stars to a specific direction. The Sgr stream mostly locates at $15-30 \mathrm{kpc}$

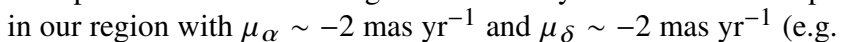
Koposov et al. 2013). We will discuss in Section 5 how the proper motion measurements are affected by the Sgr stream.

Figures 15 and 16 show the proper motion measurements for blue stars at larger distances up to $100 \mathrm{kpc}$ in the Equatorial and Galactic coordinates, respectively. The measurements at $\gtrsim 40 \mathrm{kpc}$ are noisy. After the correction of our own motion, both the velocity in the Dec. direction $\left(v_{\delta}\right)$ and in the $b$ direction $\left(v_{b}\right)$ appear to show non-zero negative values, but the significance in each distance bin is not high. More quantitatively, the averaged proper motions for all these blue stars at $\geq 40 \mathrm{kpc}$ are: $\left\langle\mu_{\alpha}\right\rangle=$

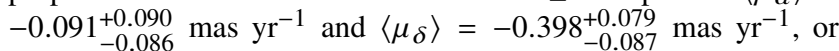
$\left\langle v_{\alpha}\right\rangle=-20.6_{-22.2}^{+23.2} \mathrm{~km} / \mathrm{s}$ and $\left\langle v_{\delta}\right\rangle=-97.1_{-23.3}^{+19.9} \mathrm{~km} / \mathrm{s}$, respectively. In the Galactic coordinate, $\left\langle\mu_{\ell} \cos b\right\rangle=-0.166_{-0.087}^{+0.083} \mathrm{mas} \mathrm{yr}^{-1}$ and

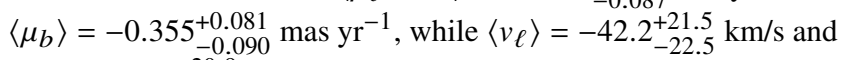
$\left\langle v_{b}\right\rangle=-87.3_{-24.4}^{+20.9} \mathrm{~km} / \mathrm{s}$, respectively. Thus the averaged proper motions between 40 and $100 \mathrm{kpc}$ indicate a perhaps $5 \sigma$-level detection of the coherent motion in the outer MW stellar halo. In Figure 16 we also show the absolute tangential velocity, $v \equiv\left[\left(v_{\ell}\right)^{2}+\left(v_{b}\right)^{2}\right]^{1 / 2}$, as a function of distances, again indicating a hint of coherent motion up to $\sim 100 \mathrm{~km} \mathrm{~s}^{-1}$. If this is genuine, it might indicate a rotation of the halo region or the existence of global motions. For example, the Large Magellanic Cloud might imprint such a motion in the halo region (e.g. Peñarrubia et al. 2016; Erkal et al. 2019; Erkal \& Belokurov 2019; Garavito-Camargo et al. 2019). However, this might be due to unknown systematics such as an imperfect correction for the DCR effect and/or a contamination of non main-sequence, faint stars such as white dwarfs (see below). We need more data to derive a more robust conclusion.

\section{DISCUSSION}

\subsection{The Sagittarius stream}

The Sgr stream is covered by our HSC-S82 footprints, and in this subsection we investigate how the proper motion measurements are affected by the stream. Within the HSC-S82 footprints, the region with R.A. $>0$ contains the $\mathrm{Sgr}$ stream, while the other with R.A. $<0$ is not affected by the Sgr stream. This can be found from 

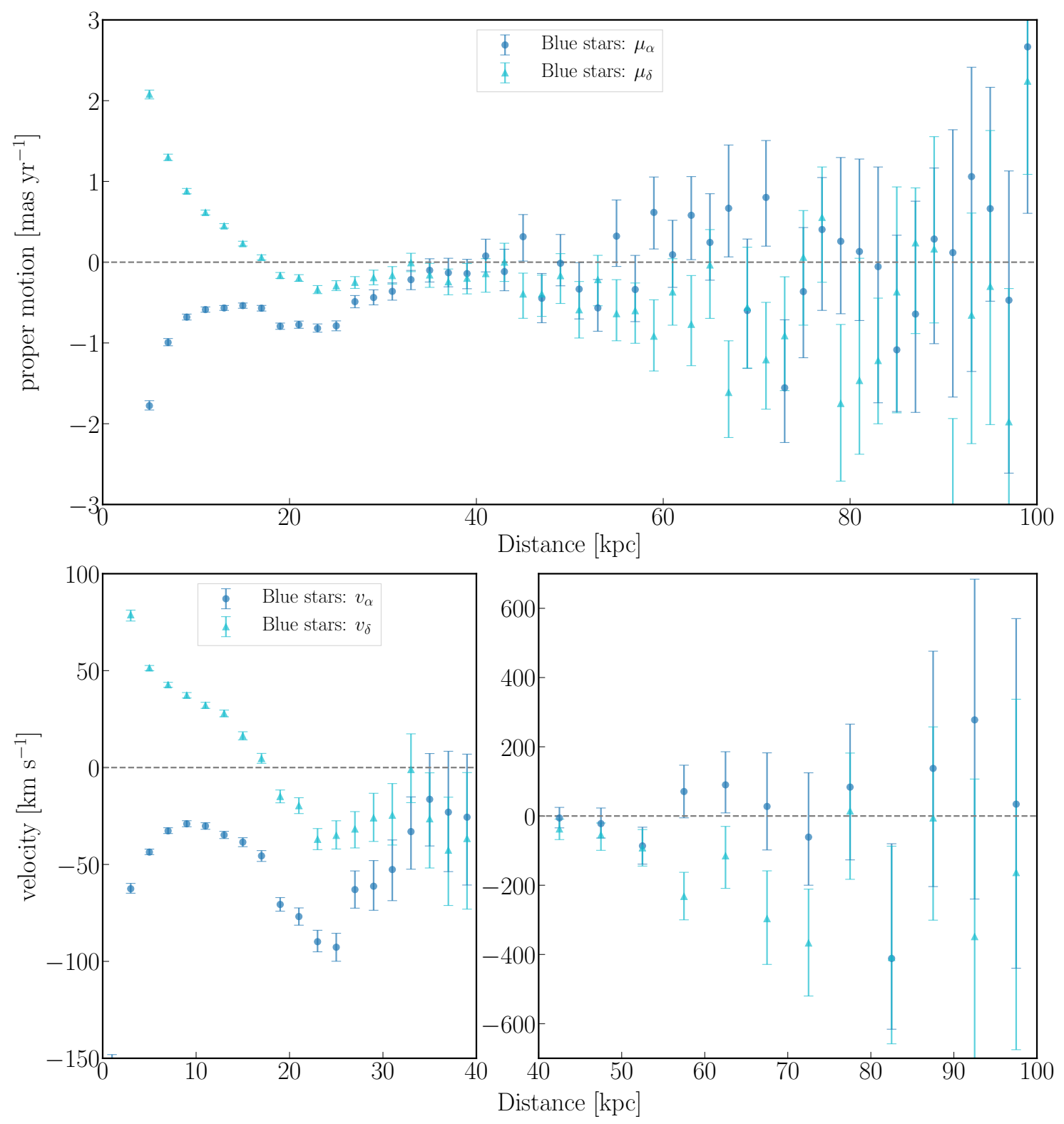

Figure 15. Similar to Figure 13, but the figure shows the component proper motions and tangential velocities for blue stars out to 100 kpc, after correcting for the effect due to our own motion. For illustrative purpose the lower two panels show the results at distances smaller and greater than 40 kpc separately, where the range of $y$-axis is adjusted to cover the range of data points. The averaged proper motions for stars at distances $\geq 40 \mathrm{kpc}^{-a r e}\left\langle\mu_{\alpha}\right\rangle=-0.091_{-0.086}^{+0.090} \mathrm{mas}^{-1}$ and $\left\langle\mu_{\delta}\right\rangle=-0.398_{-0.087}^{+0.079} \mathrm{mas} \mathrm{yr}^{-1}$, or $\left\langle v_{\alpha}\right\rangle=-20.6_{-22.2}^{+23.2} \mathrm{~km} / \mathrm{s}$ and $\left\langle v_{\delta}\right\rangle=-97.1_{-23.3}^{+19.9} \mathrm{~km} / \mathrm{s}$, respectively.

the comparison of our survey footprints (Figure 5) with the left panel of Figure 2 in Koposov et al. (2013).

Figure 17 shows a comparison between the proper motion measurements in four subregions in distances up to $40 \mathrm{kpc}$. The two subregions containing the Sgr stream at R.A. $>0$ show coherent proper motions which are negative in both $\mu_{\alpha}$ and $\mu_{\delta}$ beyond $\sim 15 \mathrm{kpc}$. This is in good agreement with the sign of the component proper motions for the Sgr stream in the S82 footprint (e.g. Koposov et al. 2013). Comparing the two subregions with the Sgr stream show that the coherent motions in the range $[15,35] \mathrm{kpc}$ look similar to each other. On the other hand, the two subregions without the Sgr stream do not display significant proper motions between 10 and $35 \mathrm{kpc}$, implying that blue stars have random motions on average in these regions. However, the results shown here could have underestimated the proper motions of Sgr tracers. If we include non-member stars in the analysis of the Sgr stream subregions and if such non-member stars do not have significant proper motions as indicated in the non-Sgr sub-regions, it would underestimate a genuine amplitude of the Sgr proper motions. Hence a further study using a cleaner sample of the Sgr tracers would be necessary, and this will be presented separately.

In Figure 18 we compare our results with the proper motions for the Gaia RR-Lyrae (RRL) stars in the two subfields with the Sgr stream (R.A.> 0) or without the Sgr stream (R.A. $<0)$ in the overlapping region of HSC and S82. RRL stars are relatively bright and have a similar intrinsic luminosity (or have a narrow distribution 

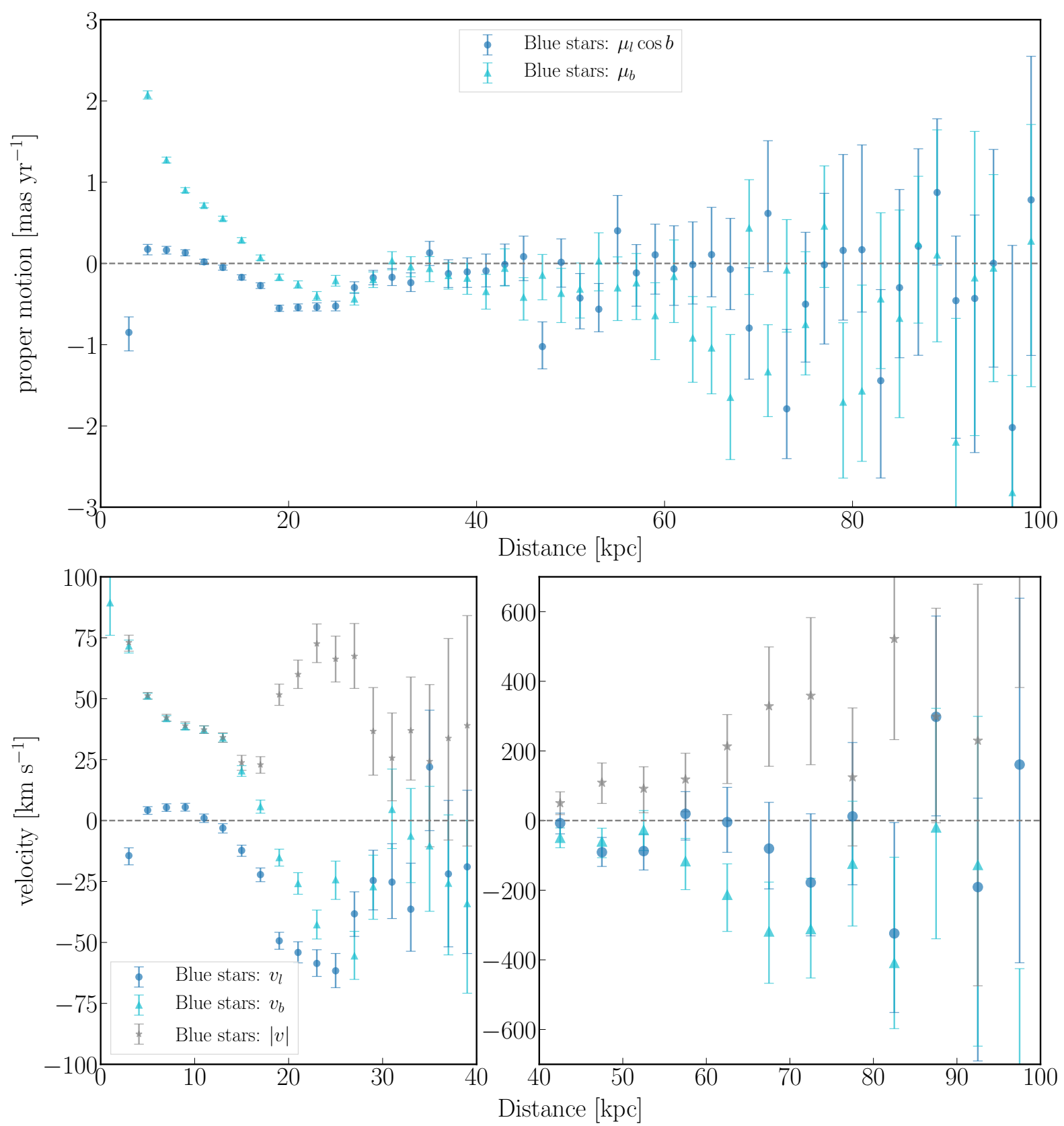

Figure 16. Similar to Figure 15, but the component proper motions and tangential velocities for blue stars are shown in the Galactic coordinates. The component tangential velocities at distances smaller and larger than $40 \mathrm{kpc}$ are shown in two separate panels, in order to more clearly demonstrate the velocity measurements on smaller distance scales. The averaged proper motions for stars at $\geq 40 \mathrm{kpc}$ are: $\left\langle\mu_{\ell} \cos b\right\rangle=-0.166_{-0.087}^{+0.083} \mathrm{mas}^{\mathrm{yr}}{ }^{-1}$ and $\left\langle\mu_{b}\right\rangle=-0.355_{-0.090}^{+0.081} \mathrm{mas}^{-1}$, while $\left\langle v_{\ell}\right\rangle=-42.2_{-22.5}^{+21.5} \mathrm{~km} / \mathrm{s}$ and $\left\langle v_{b}\right\rangle=-87.3_{-24.4}^{+20.9} \mathrm{~km} / \mathrm{s}$, respectively. We also show the tangential velocity amplitude, $|v| \equiv\left(v_{\ell}^{2}+v_{b}^{2}\right)^{1 / 2}$, as a function of distances, by the grey star symbols.

of the absolute magnitudes), so serve as useful tracers to probe the spatial structures and proper motions at relatively large distances (Antoja et al. 2020; Bellazzini et al. 2020). We identified 96 RRL stars in the HSC-S82 fields, and assume $M_{G}=0.63$ for the absolute magnitude in the Gaia filter (Muraveva et al. 2018) to estimate the distance for individual RRL. Our proper motion measurements are qualitatively in good agreement with the Gaia proper motions, for the sign and amplitudes, for both the two subfields with or without the Sgr stream. Our measurements show much more significant detections of the proper motions in this field thanks to the large statis- tics using many stars. The agreement is quite encouraging because the comparison is a totally independent test of our measurements, e.g., Gaia RRL stars are free from the DCR systematics.

\subsection{Tangential velocity dispersion}

Velocity dispersion measurements are very important for properly understanding the dynamical properties of our MW. Line-of-sight velocity dispersions have been measured in many previous studies (e.g. Xue et al. 2008; Deason et al. 2013; Kafle et al. 2014; Huang 

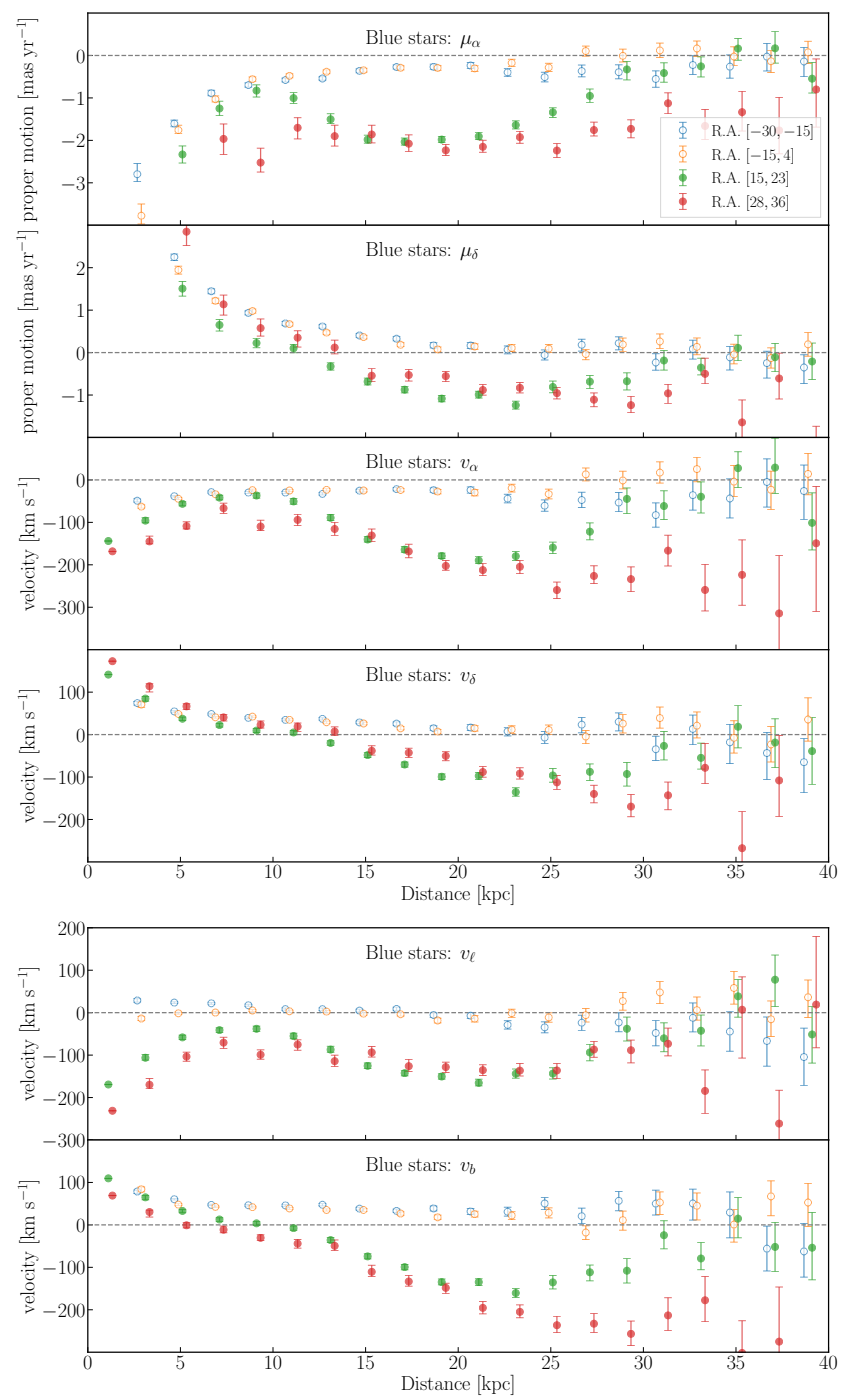

Figure 17. Similar to Figure 15, but the figure shows the component proper motions for blue stars out to $40 \mathrm{kpc}$ and for four subregions of HSC-S82 footprints, divided by the different range of R.A. $=[-30,-15],[-15,4]$, $[15,23]$ and $[28,36]$, respectively. Here the two subregions with R.A.> 0 , denoted by the filled symbols, are the regions where member stars in the Sagittarius stream are distributed, while the subregions with $\mathrm{R} . \mathrm{A}<0$, denoted by the open symbols, are not affected by the Sagittarius stream. The two panels at bottom show the velocity components, $v_{\ell}$ and $v_{b}$, in the Galactic coordinates.

et al. 2016). However, much less studies have investigated tangential velocity dispersions out to large distances due to the expenses of measuring proper motions and also because tangential velocities usually have larger measurement errors than line-of-sight velocities. In previous sections, we have provided statistical studies on the averaged proper motions and tangential velocities. With our proper motion measurements, we are also able to take a close look at the two-component tangential velocity dispersions. To measure the tangential velocity dispersions, we focus on a bright subsample of 44,502 blue stars brighter than $r_{\mathrm{S} 82, \mathrm{PSF}}=22.5$. Red stars show similar results, and thus we avoid repeatedly showing them. Stars fainter than $r_{\mathrm{S} 82, \mathrm{PSF}}=22.5$ are excluded because they have larger
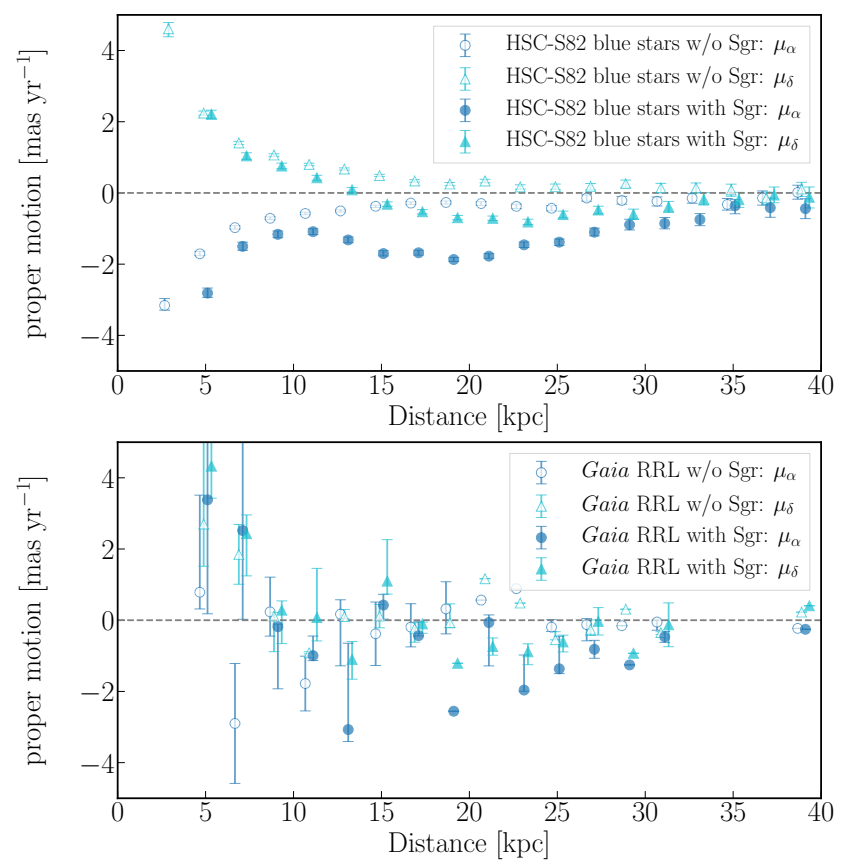

Figure 18. Comparison of the HSC-S82 proper motion measurements with RR-Lyrae proper motions from Gaia. Here we use 96 RR-Lyrae stars in the overlapping region of HSC and S82, and assumed $M_{G}=0.63$ for the absolute magnitude in the Gaia filter to estimate distances for individual RR-Lyrae stars. To increase statistics of the Gaia results, here we show the results for the two subfields, divided by R.A. $>0$ (including Sgr) or R.A. $<0$. Although the Gaia results are noisy due to low statistics, our HSC-SDSS results are qualitatively in good agreement with the Gaia results.

measurement errors, and hence properly estimating their intrinsic dispersions is more difficult.

Figure 19 shows the tangential velocity dispersions for $v_{l}$ and $v_{b}$ in the Galactic coordinates. We divide the HSC-S82 footprints into six subregions according to R.A.. Because the measurement errors dominate over the proper motion signal on individual star basis, the directly measured velocity dispersions contain both true intrinsic dispersions and the measurement errors. The measurement errors are mainly contributed by the centroid position errors from S82. However, as we will show in Appendix C, the centroid errors underestimate the true measurement errors, and thus alternatively, we estimate the measurement errors from the dispersions in apparent proper motions of quasars and small-size galaxies (e.g. Bond et al. 2010). The readers can find details in Appendix C.

The predicted measurement errors are shown as dark and light green curves in each panel of Figure 19. Following Bond et al. (2010), we choose to not subtract the measurement errors, because for our noisy measurements any small over-estimates in measurement errors can cause significant over-subtractions in true signals. To guide the eye, we also plot as red and orange dashed curves the prediction of a constant velocity dispersion of $80 \mathrm{~km} / \mathrm{s}$, combined with the measurement errors. In the top two panels, where the regions are not contaminated by the Sgr stream, our data are in good agreement with a constant velocity dispersion of $80 \mathrm{~km} / \mathrm{s}$ in the distance range 10-20 kpc. The uncertainty of this dispersion estimate increases with distance due to increasing measurement errors and is about $10 \mathrm{~km} / \mathrm{s}$ at distances below $15 \mathrm{kpc}$, and about $20 \mathrm{~km} / \mathrm{s}$ at $20 \mathrm{kpc}$. These uncertainties are dominated by distance errors at 

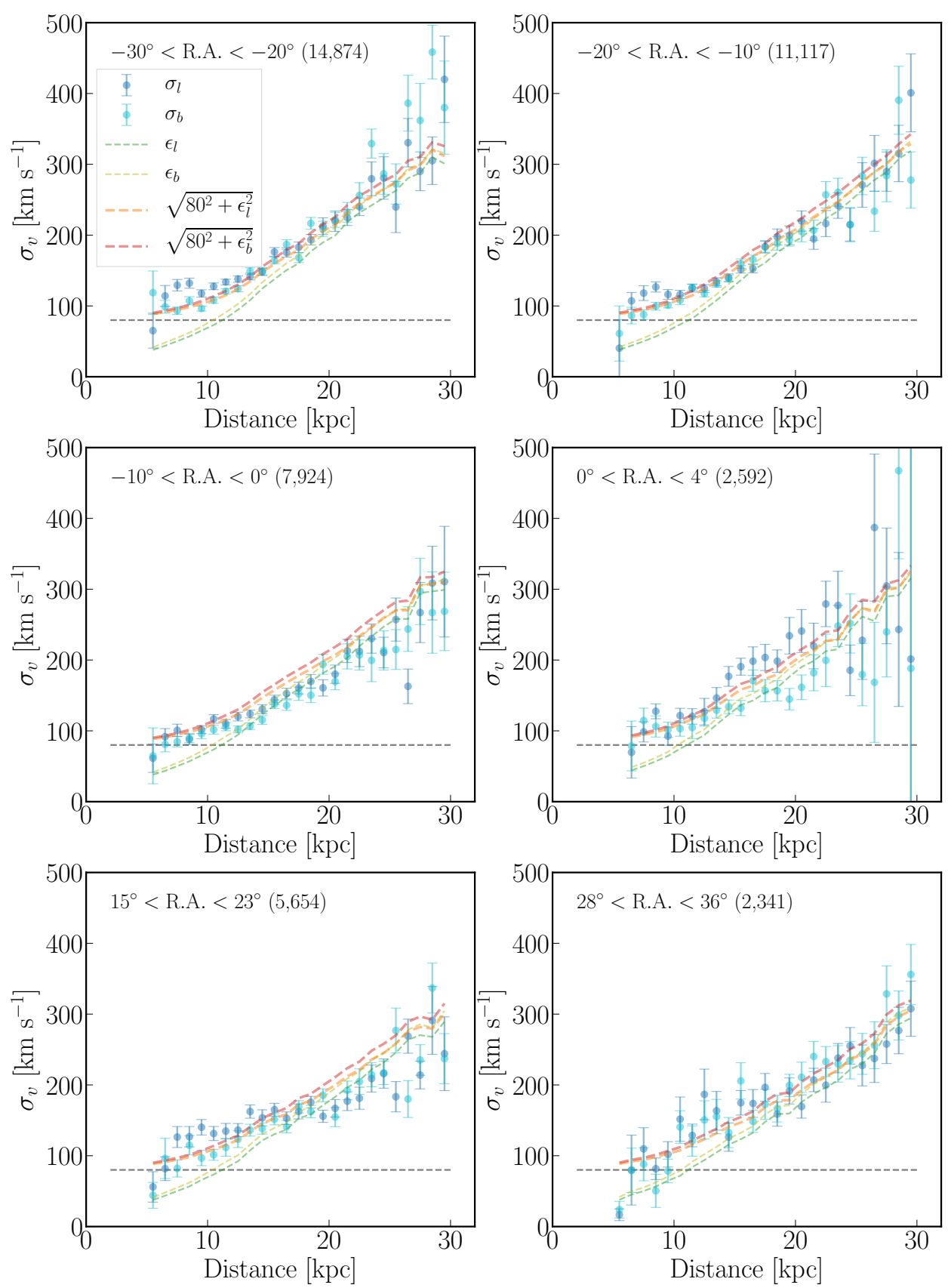

Figure 19. Tangential velocity dispersions of the two components, $v_{l}$ and $v_{b}$, in the Galactic coordinates and measured from the blue stars, for six subregions of the HSC-S 82 footprints, divided by different ranges of R.A.. The tangential velocity dispersions are contributed by both the true intrinsic velocity dispersions and the measurement errors. Dark and light green curves in each panel show the amount of measurement errors. Red and orange dashed curves show the predicted measurement for a constant velocity dispersion of $80 \mathrm{~km} / \mathrm{s}$ plus the measurement errors. The black horizontal dashed line marks the location of $80 \mathrm{~km} / \mathrm{s}$. The value in the round parentheses in each panel denotes the number of blue stars in each region. The errorbars in each bin are estimated from the 200 bootstrap realizations in the bin.

smaller distances and proper motion errors at large distances. Note that at $95 \%$ confidence level, we cannot rule out a velocity dispersion gradient of about $4.4 \mathrm{~km} / \mathrm{s} / \mathrm{kpc}$ in the distance range $10-20 \mathrm{kpc}$. The data in the other four panels also show mild agreement with a constant velocity dispersion of $80 \mathrm{~km} / \mathrm{s}$. Our measurement of the velocity dispersion for halo stars at $10 \mathrm{kpc}$ is consistent with the results from Smith et al. (2009) and Bond et al. (2010), which did not reach out to $20 \mathrm{kpc}$ though. Beyond $20 \mathrm{kpc}$, the measurement errors dominate.

The tangential velocity dispersions for $v_{l}$ and $v_{b}$ are quite similar with each other, indicating isotropic dispersions. In addition, the measured velocity dispersions are slightly smaller in the three panels with R.A.> 0, i.e., the footprints containing the Sgr stream, which is true even after considering the measurement errors. This is because the orbital motions of stars stripped from the Sgr satellite are 
correlated along the stream track. Such coherent motions decrease the velocity dispersions.

\subsection{Photometric distance errors}

As have been discussed by Jurić et al. (2008), the photometric distance estimation can have relative errors up to at most $20 \%$. In addition, the photometric error affects the photometric distance estimates. As we mentioned in Section 3.4, the median photometric errors in $g, r, i$-band are $0.0133,0.0065$ and 0.0033 mag for most of the stars thanks to the deep HSC data. Even at the very faint end (for stars with magnitude 23.5-24.5 in $i$-band), the median errors are $0.062,0.033,0.023 \mathrm{mag}$ for $g, r, i$-band. The uncertainty for $g-i$ colour which our photometric distances mostly rely on is at most 0.07 mag at the very faint end $(g, r, i \simeq 24)$. The photometric errors of 0.013 mag cause only $3 \%$ in the photometric distances on individual star basis, which is not significant compared to the errors due to uncertainty in the metallicity of stars. Fainter stars with errors of 0.07 mag could cause about $26 \%$ errors in the photometric distances, but these stars are not a majority of our sample (see Figure 3). We should note that the systematic error in distances, after the average of many stars, should be reduced by $1 / \sqrt{N}(N$ is the number of stars taken for the average in each bin). In our analysis, errors in photometric distances not only just shift results presented from Figures 12 to 19 horizontally, but it can also affect the correction for our own motion. If the photometric distances are either systematically under- or over-estimated, we might have made under- or over-corrections for our own motion. As an extreme test, even if we input $20 \%$ smaller or larger distances to correct for our own motion, it almost unchanges the results beyond $30 \mathrm{kpc}$. Within $30 \mathrm{kpc}$, increasing (or decreasing) the input distances by $20 \%$ would decrease (or increase) the proper motion measurements

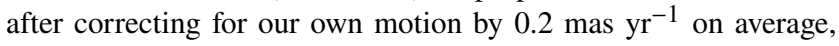
while the general trend of median proper motions as a function of distances and the relative difference between red and blue stars keep the same.

Besides the above uncertainty, we fixed the values of $[\mathrm{Fe} / \mathrm{H}]$ to -1.45 for stars at $\geq 6.25 \mathrm{kpc}$ in distances as our default analysis. As an alternative approach, we have tried to assign each blue star with a value of $[\mathrm{Fe} / \mathrm{H}]$ drawn randomly from the Gaussian distribution with $\langle[\mathrm{Fe} / \mathrm{H}]\rangle=-1.45$ and $\sigma=0.41 \mathrm{dex}$. The Gaussian distribution is based on the real $[\mathrm{Fe} / \mathrm{H}]$ distribution of blue stars (see Figure 10 in Ivezić et al. 2008). We confirmed that the randomly assigned metallicity leads to a small change by less than $0.1{\text { mas } \mathrm{yr}^{-1}}^{-1}$ in our averaged proper motion measurements at fixed distances. On the other hand, because some blue stars in the inner stellar halo are found to have extreme values of $[\mathrm{Fe} / \mathrm{H}$ ] as low as -2 (Carollo et al. 2007; de Jong et al. 2010; An et al. 2013; An \& Beers 2019; Dietz et al. 2019, e.g.), as an extreme test, we fix $[\mathrm{Fe} / \mathrm{H}]=-0.95$ or -1.95 , i.e., shifted from the fiducial value by \pm 0.5 , for all red and blue stars beyond $6.25 \mathrm{kpc}$, it causes roughly $20 \%$ or $-20 \%$ shifts in photometric distances, but both cause mainly a horizontal shift in the proper motion profile against distances at $\gtrsim 10 \mathrm{kpc}$. For nearby blue stars within $6.25 \mathrm{kpc}$, we calculated their metallicity based on their $(u-g)$ and $(g-r)$ colours for results in the main text. In this way, their mean metallicity is about $[\mathrm{Fe} / \mathrm{H}] \sim-1.2$. After fixing their $[\mathrm{Fe} / \mathrm{H}]$ to -1.95 , the proper motions after correcting for our own motion change by about $25 \%$, while our main conclusions are not affected. Hence our results are qualitatively valid. Nevertheless a further careful study, e.g. using spectroscopic observations for such distant red stars, would be needed to understand the impact of metallicities for halo stars.

\subsection{Contamination by non-main-sequence stars}

The focus of this paper is to measure and investigate the proper motions of main-sequence stars. Moreover, our approach of photometric distance estimates are based on the luminosity-colour relation of main-sequence stars. Hence, if our star sample is contaminated by other types of non-main-sequence stars, our estimated photometric distances and thus the results from Figures 12 to 19 might be affected, even though main stars are a majority population of stellar objects.

If our star sample is contaminated by giants, these intrinsically bright giants treated as fainter main-sequence stars can cause underestimates in photometric distances and thus over-corrections of our own motion. Jurić et al. (2008) have removed stars scattered by 0.3 magnitudes away from the stellar locus in colour-colour space for main-sequence stars, which helped to eliminate the contamination by giants. In addition, Jurić et al. (2008) built a fiducial Galaxy model to investigate the contamination by giants in their sample, and they reported a worst fraction of about $5 \%$. In our analysis, the HSC flux and colour are more precise than those of SDSS, and we have applied 3- $\sigma$ clipping to our sample of stars in colour-colour space. The contamination by giants in our analysis is not expected to be higher than that of Jurić et al. (2008). Furthermore, we have repeated our analysis by adopting 1- $\sigma$ clipping to our sample of stars in colour-colour space, to throw away even more stars scattered away from the main-sequence stellar locus, and our results presented from Figures 12 to 19 are proved to be robust against different levels of $\sigma$ clippings. We have also looked at the number density profile for our sample of stars as a function of $z$ distance to the disc plane, and there is no indication of flattening that can be considered as a sign of the contamination by red giants. In the end, we emphasise that giant stars are usually more distant and hence have smaller observed proper motions, the inclusion of a small fraction of giant stars in our sample are thus not expected to significantly change our median/mean proper motion measurements.

On the other hand, if our star sample is contaminated by intrinsically faint and blue non-main-sequence stars, such as white dwarfs, these intrinsically faint white dwarfs treated as brighter main-sequence stars can cause over-estimates in photometric distances and hence under-corrections for our own motion. In addition, white dwarfs are usually observed in nearby distances, and thus have large observed proper motions. If our sample of stars is contaminated by white dwarfs, the median/mean proper motion measurements might be biased to be larger. However, we believe that a contamination of white dwarfs to our sample is small, as described in the following. First of all, the total number of white dwarfs is only about $10 \%$ of the number of main-sequence stars as predicted by the standard MW model (also see Mróz et al. 2017; Niikura et al. 2019, for the microlensing observations). Figure 20 shows the distribution of white dwarfs in the colour-colour diagram of $g-r$ vs. $g-i$, compared to that of main-sequence stars of the sample used in our proper motion measurements. For this comparison, we use a secure sample of white dwarfs that is taken from the SDSS DR7 spectroscopic sample of white dwarfs (Kleinman et al. 2013). The figure shows that only about $3.5 \%$ of white dwarfs lie into the region of colours which we use to define our sample of main-sequence stars (after including 3- $\sigma$ clipping around the locus of main-sequence stars). Hence we conclude that white dwarfs, even if exist, give only about $0.35 \%(=0.1 \times 0.035)$ contamination to our sample (only for the blue star sample). Although we think that our results are unlikely significantly affected by the contamination of white dwarfs, the possible contamination could affect the proper 


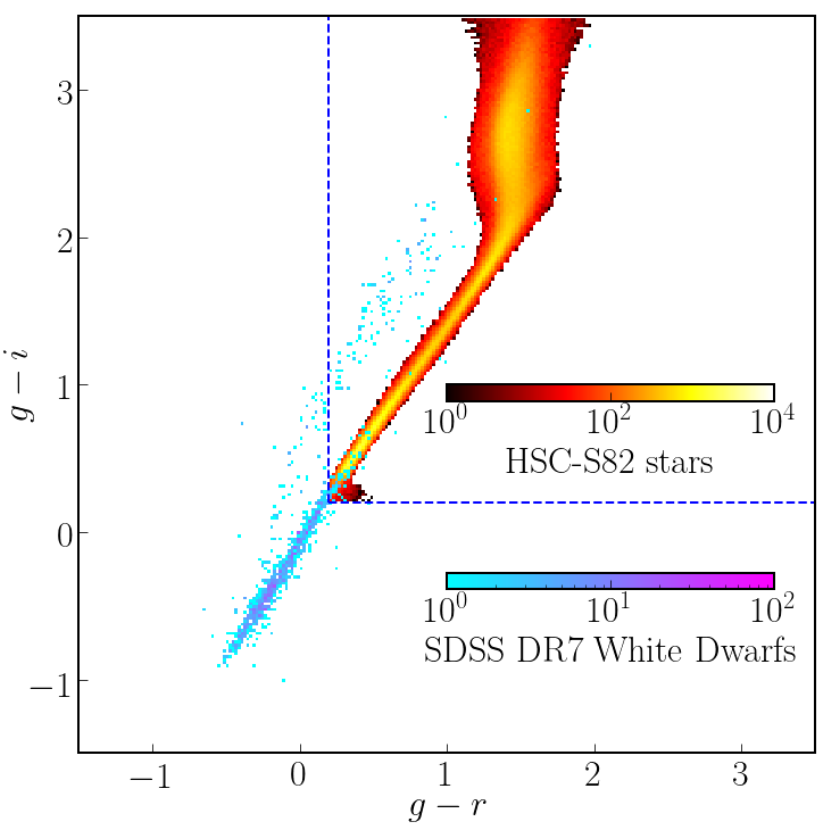

Figure 20. The distribution of main-sequence stars used in our fiducial analysis in the colour-colour diagram of $(g-r)$ vs. $(g-i)$, compared to that of white dwarfs that are taken from the SDSS DR7 spectroscopic catalog of white dwarfs. The colours in each pixel denote the number of stars or white dwarfs in the pixel. Note that the main-sequence stars are after the 3- $\sigma$ clipping around the locus of main-sequence stars in the colour space, and $g-r$ and $g-i$ are the colours used for photometric distance estimation of each star. The blue dashed lines denote the colour cuts that we use to define our sample of main-sequence stars. Most of white dwarfs are outside the colour cuts, and only $3.5 \%$ of white dwarfs lie into the colour region of main-sequence stars.

motion measurements at large distances, especially $d>40 \mathrm{kpc}$, where we found an apparent non-zero proper motion. This requires a more careful study, and is our future study by using more datasets. In addition, searching for white dwarfs themselves from our deep photometry and proper motion measurements, e.g. through hyper velocities, would be interesting, and this will be our future study.

\section{CONCLUSION}

By taking advantage of the nearly 12 years of time baseline leverage between SDSS and HSC, we measure the proper motions for more than 0.55 million stars. Thanks to the superb image quality and the deep coadd photometric sources from SDSS Stripe 82 (S82) and HSC, we are able to make proper motion measurements for stars down to $\sim 24$ in $i$-band, which is 3 magnitudes deeper than the nominal SDSS depth, and is deeper than almost all of the previous proper motion catalogues. The net proper motions of stars, including the contribution of the Galactic rotation, can be measured out to $100 \mathrm{kpc}$. In particular the use of HSC data was crucial for our study; for example, the HSC data allows for a secure identification of stars from the matched catalogues.

We adopt the mean epoch difference for each individually matched source between S82 and HSC as its time baseline, to calculate the proper motion in unit of mas $\mathrm{yr}^{-1}$. The matched galaxies are used to recalibrate systematic errors in astrometry and to bring the observations taken at different epochs to be in the same reference frame.

We first compare our measured proper motions with the Gaia DR2 proper motions, which shows very good overall agreement. The median systematic bias of our proper motion measurements only differ from Gaia by at most $0.052 \mathrm{mas} \mathrm{yr}^{-1}$ (see Figure D1). The scatter is mainly contributed by the proper motion errors from Gaia itself, because the matched stars are at faint end of the Gaia catalogue, $20<G<21$, where the Gaia proper motion errors are relatively large.

However, after dividing stars into red and blue populations, each of them shows a non-negligible median offset from Gaia DR2 proper motions in $\mu_{\delta}$, which is in good agreement with the effect of differential chromatic diffraction (DCR) by the Earth's atmosphere for the SDSS images, where the Gaia space observation is free from this kind of systematics and the effect in the HSC images is corrected by the Atmospheric Dispersion Compensator. The offsets are $\Delta \mu_{\delta} \simeq$

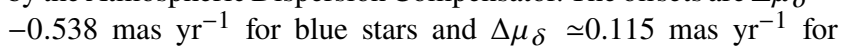
red stars, respectively. In addition, an independent test using the matched quasars, which have bluer spectra from galaxies, reveal

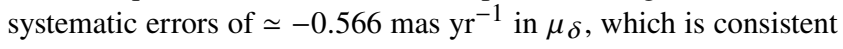
with the DCR effect in comparison with the Gaia results. Hence, as our default analysis method, we corrected for the DRC effects for individual stars using the measured offsets in the Gaia comparison as a function of colours of stars $(g-i)$. This DCR correction is

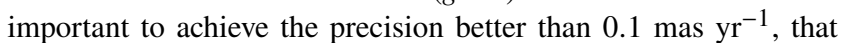
is needed to study the proper motions at large distances in the Galactic halo region after subtracting the contribution due to own own motion.

We estimate the photometric distance of individual stars, following the method in Jurić et al. (2008) and Ivezić et al. (2008). The directly measured proper motions show coherent R.A. and distance dependent motions, most of which are due to our own motion (the Galactic rotation plus the Sun's motion with respect to the Local Standard of Rest). After correcting for our own motion, we find the proper motions are dominated by the Galactic disc rotation within $1 \mathrm{kpc}$, and gradually decrease out to $5 \mathrm{kpc}$. Our measurements are affected by the Sgr stream, which have negative proper motions in both $\mu_{\alpha}$ and $\mu_{\delta}$ beyond $13 \mathrm{kpc}$. By looking into the footprint without the Sgr stream, we do not find any significant coherent proper motions in the distance range between 10 and $40 \mathrm{kpc}$ (Figures 14 and 17). The kinematical structures in the halo region are useful to study the assembly history of the MW, and a further careful study including properties of the Sgr stream will be presented elsewhere. As a prospect for future observation, the Subaru Prime Focus Spectrograph (Takada et al. 2014), which is currently under construction, would be powerful to study radial motions and chemical compositions for distant stars that provide very complementary information to the proper motions and other information from imaging datasets.

Beyond $40 \mathrm{kpc}$, the measurements are noisy, but the motions of blue stars in the more distant stellar halo are close to random. After adopting wider bins in distance, we find the velocity in the Dec. direction $\left(v_{\delta}\right)$ or in the $b$ direction $\left(v_{b}\right)$ both appears to show non-zero negative values, but the significance in each distance bin is not high due to the limited statistics. If this is genuine, it might indicate the existence of global motions in the distant stellar halo, but we need more data to reach a rigorous conclusion.

The two-component tangential velocity dispersions are measured for a subsample of blue stars brighter than $r_{\mathrm{S} 82, \mathrm{PSF}}=22.5$. Following Bond et al. (2010), we estimate the measurement errors through the dispersion in apparent proper motions of small-size galaxies and quasars after astrometry recalibrations to remove the 
spatial variations of astrometric systematics, i.e., the middle panel of Figure 5. We measured the tangential velocity dispersion in the distance range 5-20 kpc and find that the data are consistent with a constant isotropic dispersion of $80 \pm 10 \mathrm{~km} / \mathrm{s}$, which is consistent with the earlier works (Smith et al. 2009; Bond et al. 2010) for the shorter distances of $<10 \mathrm{kpc}$. The footprints containing the Sgr stream tend to show slightly smaller velocity dispersions, which is due to the correlation in orbital motions of stripped stars from the Sgr dwarf.

Tangential velocity dispersions are crucial for properly constraining the velocity anisotropy of halo stars. Early studies circumvent the unknown anisotropy by adopting arbitrary values, treating it as a free but constant parameter, assuming a certain functional form, constraining it from numerical simulations or modelling the velocity ellipsoid (e.g. Battaglia et al. 2005; Dehnen et al. 2006; Xue et al. 2008; Watkins et al. 2010; Kafle et al. 2014). These approaches, however, unavoidably introduce additional uncertainties (Wang et al. 2018). The situation has been significantly improved by the advent of Gaia proper motions for bright halo stars, globular clusters and satellite galaxies (e.g. Sohn et al. 2018; Watkins et al. 2019; Li et al. 2019, 2020; Callingham et al. 2019; Posti \& Helmi 2019; Vasiliev 2019; Eadie \& Jurić 2019; Fritz et al. 2020). However, Gaia proper motions for individual main-sequence stars are still confined within very nearby distances. The tangential velocity dispersion for our sample of main-sequence stars is thus very helpful. Although currently we do not have line-of-sight velocities, by assuming the velocity anisotropy of different stellar populations are similar to each other, we can combine existing line-of-sight velocity dispersion measurements from other types of stars to constrain the anisotropy of the stellar halo. However, such an assumption still awaits further tests, and we leave more detailed discussions to a future study.

Our results prove the power of statistical method for measuring proper motions of distant stars, especially in the halo regions of the MW. In this paper we mainly focus on the averaged proper motions of stars and their dispersions, and did not focus on rare populations of the proper motion distribution in a given bin or the spatial structures of the proper motions. For example, a tail population such as hyper-velocity stars is a useful tracer of the gravitational potential strength of the MW, and the spatial structures of the proper motions are powerful to study the substructure remnants and assembly histories of the MW. These are our future work, and will be presented elsewhere as well. Our method can be applied to ongoing and upcoming surveys such as the DES, LSST, Euclid and WFIRST. The current study is the first step of the statistical proper motion measurements, and we hope our work presented in this paper gives a guidance for these surveys.

\section{ACKNOWLEDGEMENTS}

The Hyper Suprime-Cam (HSC) collaboration includes the astronomical communities of Japan and Taiwan, and Princeton University. The HSC instrumentation and software were developed by the National Astronomical Observatory of Japan (NAOJ), the Kavli Institute for the Physics and Mathematics of the Universe (Kavli IPMU), the University of Tokyo, the High Energy Accelerator Research Organization (KEK), the Academia Sinica Institute for Astronomy and Astrophysics in Taiwan (ASIAA), and Princeton University. Funding was contributed by the FIRST program from Japanese Cabinet Office, the Ministry of Education, Culture, Sports, Science and Technology (MEXT), the Japan Society for the Pro- motion of Science (JSPS), Japan Science and Technology Agency (JST), the Toray Science Foundation, NAOJ, Kavli IPMU, KEK, ASIAA, and Princeton University.

This paper makes use of software developed for the Large Synoptic Survey Telescope. We thank the LSST Project for making their code available as free software at http://dm. lsst.org.

The Pan-STARRS1 Surveys (PS1) and the PS1 public science archive have been made possible through contributions by the Institute for Astronomy, the University of Hawaii, the Pan-STARRS Project Office, the Max-Planck Society and its participating institutes, the Max Planck Institute for Astronomy, Heidelberg and the Max Planck Institute for Extraterrestrial Physics, Garching, The Johns Hopkins University, Durham University, the University of Edinburgh, the Queen's University Belfast, the Harvard-Smithsonian Center for Astrophysics, the Las Cumbres Observatory Global Telescope Network Incorporated, the National Central University of Taiwan, the Space Telescope Science Institute, the National Aeronautics and Space Administration under Grant No. NNX08AR22G issued through the Planetary Science Division of the NASA Science Mission Directorate, the National Science Foundation Grant No. AST-1238877, the University of Maryland, Eotvos Lorand University (ELTE), the Los Alamos National Laboratory, and the Gordon and Betty Moore Foundation.

This paper is based on data collected at the Subaru Telescope and retrieved from the HSC data archive system, which is operated by Subaru Telescope and Astronomy Data Center at National Astronomical Observatory of Japan. Data analysis was in part carried out with the cooperation of Center for Computational Astrophysics, National Astronomical Observatory of Japan.

We would like to thank Jim Annis, Ana Bonaca, Linghua Jiang, Chervin Laporte, Surhud More, Xiangchong Li, Hu Zou, Xu Zhou, Zhaoyu Li and Chao Liu for useful discussions and helps. This work is supported in part by World Premier International Research Center Initiative (WPI Initiative), MEXT, Japan, and JSPS KAKENHI Grant Numbers JP15H03654, JP15H05887, JP15H05893, JP15H05896, JP15K21733, and JP19H00677. Wenting Wang is supported by NSFC (12022307).

\section{DATA AVAILABILITY}

The data underlying this article are available in HSCSSP, at https://doi.org/10.1093/pasj/psz103, SDSS DR7 Stripe82 at https://doi.org/10.1088/0004-637X/794/2/120, Gaia DR2 at https://doi.org/10.1051/0004-6361/201833051 and SDSS DR14 quasar at https://doi.org/10.1051/0004-6361/201732445. The datasets were derived from sources in the public domain: HSC-SSP https://hsc-release.mtk.nao.ac.jp/doc/, SDSS Catalogue Archive Server http://skyserver.sdss.org/CasJobs/, Gaia Archive https://gea.esac.esa.int/archive/ and SDSS Quasar Catalogue https://www.sdss.org/dr14/algorithms/qso_catalog/.

\section{REFERENCES}

Abazajian K., Adelman-McCarthy J. K., Agüeros M. A., Allam S. S., Anderson S. F., Annis J., Bahcall N. A., Baldry I. K., 2003, AJ, 126, 2081

Abazajian K., et al., 2004, AJ, 128, 502

Aihara H., Arimoto N., Armstrong R., Arnouts S., Bahcall N. A., Bickerton S., Bosch J., Bundy K., 2018a, PASJ, 70, S4

Aihara H., et al., 2018b, PASJ, 70, S8

Aihara H., et al., 2019, PASJ, p. 106 
An D., Beers T. C., 2019, arXiv e-prints, p. arXiv:1912.06847

An D., et al., 2013, ApJ, 763, 65

Annis J., et al., 2014, ApJ, 794, 120

Antoja T., Ramos P., Mateu C., Helmi A., Anders F., Jordi C., Carballo-Bello J. A., 2020, A\&A, 635, L3

Battaglia G., et al., 2005, MNRAS, 364, 433

Bellazzini M., Ibata R., Malhan K., Martin N., Famaey B., Thomas G., 2020, arXiv e-prints, p. arXiv:2003.07871

Bonaca A., Hogg D. W., Price-Whelan A. M., Conroy C., 2019, ApJ, 880, 38

Bond N. A., et al., 2010, The Astrophysical Journal, 716, 1

Bosch J., et al., 2018, PASJ, 70, S5

Boylan-Kolchin M., Bullock J. S., Kaplinghat M., 2011, MNRAS, 415, L40

Bramich D. M., et al., 2008, Monthly Notices of the Royal Astronomical Society, 386, 887

Callingham T. M., et al., 2019, MNRAS, 484, 5453

Carollo D., et al., 2007, Nature, 450, 1020

Cautun M., Bose S., Frenk C. S., Guo Q., Han J., Hellwing W. A., Sawala T., Wang W., 2015, MNRAS, 452, 3838

Chambers K. C., et al., 2016, arXiv e-prints, p. arXiv:1612.05560

Deason A. J., Van der Marel R. P., Guhathakurta P., Sohn S. T., Brown T. M., 2013, ApJ, 766, 24

Dehnen W., McLaughlin D. E., Sachania J., 2006, MNRAS, 369, 1688

Dietz S. E., Yoon J., Beers T. C., Placco V. M., 2019, arXiv e-prints, p. arXiv: 1911.11140

Eadie G., Jurić M., 2019, ApJ, 875, 159

Erkal D., Belokurov V. A., 2019, arXiv e-prints, p. arXiv:1907.09484

Erkal D., et al., 2019, MNRAS, 487, 2685

Filippenko A. V., 1982, PASP, 94, 715

Fritz T. K., Di Cintio A., Battaglia G., Brook C., Taibi S., 2020, arXiv e-prints, p. arXiv:2001.02651

Fukugita M., Ichikawa T., Gunn J. E., Doi M., Shimasaku K., Schneider D. P., 1996, AJ, 111, 1748

Furusawa H., et al., 2018, PASJ, 70, S3

Gaia Collaboration et al., 2016, A\&A, 595, A1

Gaia Collaboration et al., 2018, A\&A, 616, A1

Gallart C., Bernard E. J., Brook C. B., Ruiz-Lara T., Cassisi S., Hill V., Monelli M., 2019, Nature Astronomy, 3, 932

Garavito-Camargo N., Besla G., Laporte C. F. P., Johnston K. V., Gómez F. A., Watkins L. L., 2019, ApJ, 884, 51

Gould A., Kollmeier J. A., 2004, ApJS, 152, 103

Gunn J. E., et al., 1998, AJ, 116, 3040

Gunn J. E., et al., 2006, AJ, 131, 2332

Hambly N. C., et al., 2001a, MNRAS, 326, 1279

Hambly N. C., Davenhall A. C., Irwin M. J., MacGillivray H. T., 2001b, MNRAS, 326, 1315

Helmi A., 2020, arXiv e-prints, p. arXiv:2002.04340

Helmi A., Babusiaux C., Koppelman H. H., Massari D., Veljanoski J., Brown A. G. A., 2018, Nature, 563, 85

Høg E., et al., 2000, A\&A, 355, L27

Huang Y., et al., 2016, MNRAS, 463, 2623

Ivezić Ž., et al., 2008, ApJ, 684, 287

Ivezić Ž., Connolly A., Vanderplas J., Gray A., 2014, Statistics, Data Mining and Machine Learning in Astronomy. Princeton University Press

Ivezić Ž., Kahn S. M., Tyson J. A., Abel B., Acosta E., Allsman R., Alonso D., AlSayyad Y., 2019, ApJ, 873, 111

Jiang L., et al., 2014, ApJS, 213, 12

Jurić M., et al., 2008, ApJ, 673, 864

Jurić M., et al., 2017, The LSST Data Management System. p. 279

Kafle P. R., Sharma S., Lewis G. F., Bland-Hawthorn J., 2014, ApJ, 794, 59

Kawanomoto S., et al., 2018, PASJ, 70, 66

Kleinman S. J., et al., 2013, ApJS, 204, 5

Klypin A., Kravtsov A. V., Valenzuela O., Prada F., 1999, ApJ, 522, 82

Komiyama Y., et al., 2018, PASJ, 70, S2

Koposov S. E., Belokurov V., Evans N. W., 2013, ApJ, 766, 79

LSST Science Collaboration Marshall P., Anguita T., Bianco F. B., Bellm E. C., Brandt N., Clarkson W., Connolly A., 2017, arXiv e-prints, p. arXiv: 1708.04058
Laporte C. F. P., Johnston K. V., Gómez F. A., Garavito-Camargo N., Besla G., 2018, MNRAS, 481, 286

Lépine S., Shara M. M., Rich R. M., 2003, AJ, 126, 921

Li Z.-Z., Qian Y.-Z., Han J., Wang W., Jing Y. P., 2019, ApJ, 886, 69

Li Z.-Z., Qian Y.-Z., Han J., Li T. S., Wang W., Jing Y. P., 2020, ApJ, 894, 10

Luyten W. J., Hughes H. S., 1980, Proper Motion Survey, University of Minnesota, 55, 1

Magnier E. A., et al., 2013, ApJS, 205, 20

Magnier E. A., et al., 2016, arXiv e-prints, p. arXiv:1612.05242

Mandelbaum R., et al., 2018, PASJ, 70, S25

Miyazaki S., et al., 2018, PASJ, 70, S1

Monet D. G., et al., 2003, AJ, 125, 984

Moore B., Ghigna S., Governato F., Lake G., Quinn T., Stadel J., Tozzi P., 1999, ApJ, 524, L19

Mróz P., et al., 2017, Nature, 548, 183

Munn J. A., et al., 2004, AJ, 127, 3034

Munn J. A., et al., 2014, AJ, 148, 132

Muraveva T., Delgado H. E., Clementini G., Sarro L. M., Garofalo A., 2018, MNRAS, 481, 1195

Niikura H., Takada M., Yokoyama S., Sumi T., Masaki S., 2019, Phys. Rev. D, 99, 083503

Pâris I., et al., 2018, A\&A, 613, A51

Peñarrubia J., Gómez F. A., Besla G., Erkal D., Ma Y.-Z., 2016, MNRAS, 456, L54

Pier J. R., Munn J. A., Hindsley R. B., Hennessy G. S., Kent S. M., Lupton R. H., Ivezić Ž., 2003, AJ, 125, 1559

Posti L., Helmi A., 2019, A\&A, 621, A56

Ruiz M. T., Wischnjewsky M., Rojo P. M., Gonzalez L. E., 2001, ApJS, 133, 119

Schlafly E. F., et al., 2012, ApJ, 756, 158

Schönrich R., Binney J., Dehnen W., 2010, MNRAS, 403, 1829

Smith M. C., et al., 2009, MNRAS, 399, 1223

Sohn S. T., Watkins L. L., Fardal M. A., van der Marel R. P., Deason A. J., Besla G., Bellini A., 2018, ApJ, 862, 52

Takada M., et al., 2014, PASJ, 66, R1

Tian H.-J., et al., 2017, ApJS, 232, 4

Tonry J. L., et al., 2012, ApJ, 750, 99

Vasiliev E., 2019, MNRAS, 484, 2832

Wang W., Han J., Cole S., More S., Frenk C., Schaller M., 2018, MNRAS, 476, 5669

Wang W., Han J., Cautun M., Li Z., Ishigaki M. N., 2019, arXiv e-prints, p. arXiv: 1912.02599

Watkins L. L., Evans N. W., An J. H., 2010, MNRAS, 406, 264

Watkins L. L., van der Marel R. P., Sohn S. T., Evans N. W., 2019, ApJ, 873, 118

Xue X. X., et al., 2008, ApJ, 684, 1143

York D. G., et al., 2000, AJ, 120, 1579

Zacharias N., et al., 2000, AJ, 120, 2131

de Boer T. J. L., Belokurov V., Koposov S. E., 2018, MNRAS, 473, 647

de Jong J. T. A., Yanny B., Rix H.-W., Dolphin A. E., Martin N. F., Beers T. C., 2010, ApJ, 714, 663

\section{APPENDIX A: QUERY FOR SAMPLES IN HSC AND S82}

In the following, we provide the queries that we adopted to download our sample of sources from HSC (the HSC-SSP Public Data Release webpage $^{10}$ ) and S82 (SDSS catalog Archive Server ${ }^{11}$ ).

10 https://hsc-release.mtk.nao.ac.jp/doc/

11 http://skyserver.sdss.org/CasJobs/ 


\section{A1 HSC}

\section{SELECT}

object_id, ra, dec, i_extendedness_value,

i_sdsscentroid_ra, i_sdsscentroid_dec,

i_sdsscentroid_rasigma, i_sdsscentroid_decsigma,

g_cmodel_mag, $r_{-}$cmodel_mag,

i_cmodel_mag, $z_{-}$cmodel_mag,

g_cmodel_magsigma, r_cmodel_magsigma,

i_cmodel_magsigma, z_cmodel_magsigma

g_psfflux_mag, r_psfflux_mag,

i_psfflux_mag, z_psfflux_mag,

g_psfflux_magsigma, r_psfflux_magsigma,

i_psfflux_magsigma, z_psfflux_magsigma

FROM

s18a_wide.forced

LEFT JOIN

s18a_wide.forced2 USING (object_id)

WHERE

i_psfflux_mag $>17$ and i_psfflux_mag $<24.5$

and $\operatorname{dec}>-1.26$ and $\operatorname{dec}<1.26$ and ( $r a<=60$ or $r a>=300$ )

and isprimary

\section{A2 $\mathbf{5 8 2}$}

\section{SELECT}

objID, ra, dec,

run, rerun, camcol, field, flags, type,

rowc_r, colc_r, rowcErr_r, colcErr_r,

$u, g, r, i, z$,

err_u, err_g, err_r, err_i, err_z,

psfmag_u, psfMag_g, psfMag_r, psfMag_i, psfMag_z, psfmagerr_u,psfmagerr_g, psfmagerr_r, psfmagerr_i, psfmagerr_z

into mydb.all from PhotoPrimary

WHERE

$(($ flags \& $0 \times 10000000) \quad !=0)$

AND ( (flags \& $0 \times 8100000 c 00 a 4)=0)$

AND $(((f$ lags \& $0 \times 400000000000)=0)$ or

(psfmagerr_r $<=0.2$ and psfmagerr $i<<=0.2$

and psfmagerr_g $<=0.2)$ )

AND $(($ flags \& $0 \times 100000000000)=0)$ or (flags \&

$0 \times 1000)=0)$

AND (run $=106$ or run $=206$ )

AND ( (ra BETWEEN 329 and 360) or (ra BETWEEN 0 and 36))

AND (psfMag_i BETWEEN 17 and 24.2)

\section{APPENDIX B: THE IMPORTANCE OF ASTROMETRY RECALIBRATION AND PROPER ESTIMATES OF TIME BASELINES}

Throughout the main text of the paper, we measure proper motions for main-sequence stars by comparing the R.A. and Dec. coordinates between matched photometric sources detected on deep coadd images from the SDSS Stripe 82 region and HSC. We estimate the time baseline from the mean epoch difference based on all single exposure images adopted for coadding. On the other hand, galaxies have been used to recalibrate the astrometry of stars, which has helped to correct for residual systematics in astrometry and set up the common reference frame.
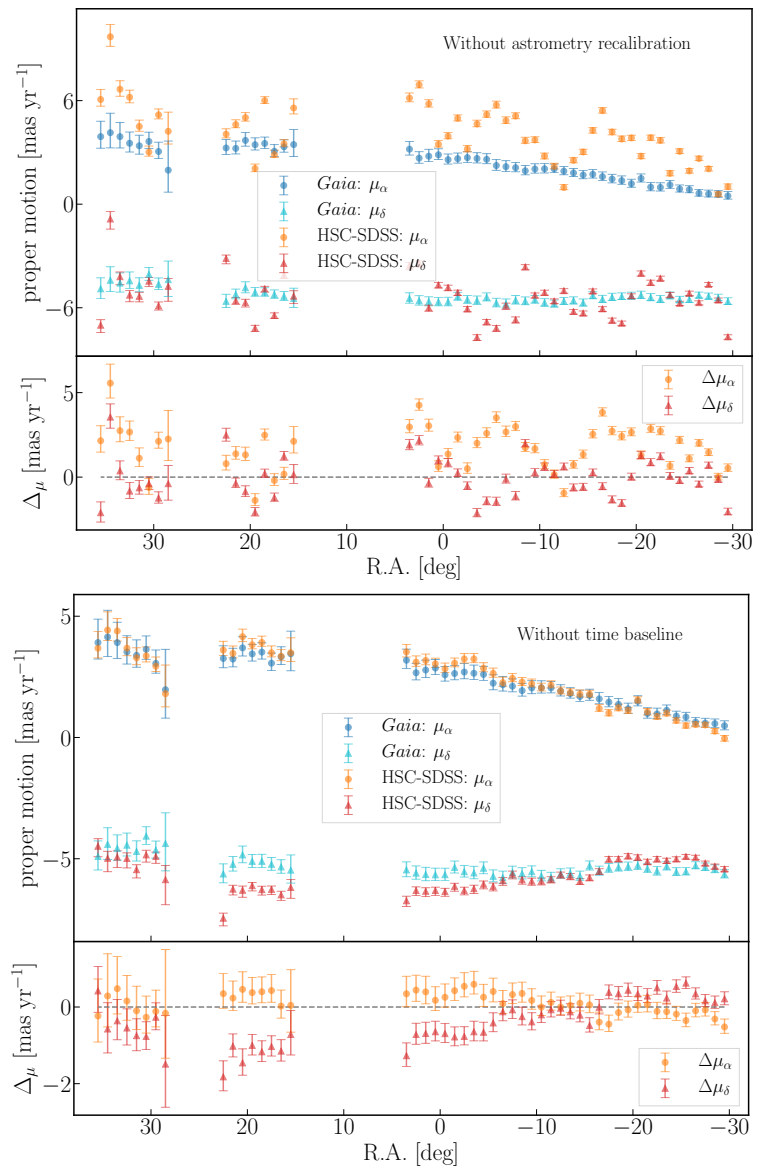

Figure B1. Similar to Figure 7, but here we show the proper motion measurements when either the astrometry recalibration is not implemented or the time baseline is not correctly estimated. Upper panels: The results without the astrometry recalibration using the angular offsets of galaxies between HSC and S82 (see Section 3.3.1), but with the correctly estimated time baseline. If we do not use this recalibration, the HSC-S82 proper motions display

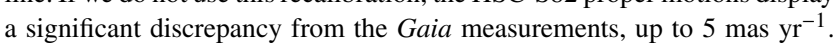
Lower: The results if we fix 12 years for the HSC-S82 time baseline in the proper motion estimations, rather than using the more exact mean epoch difference for each star in Figure 1. The fixed 12-years of time baseline

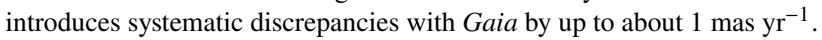

Figure B1 demonstrates that both astrometry recalibration using galaxies and proper estimates of time baselines are very important. If we do not include the astrometry recalibration, the measured proper motions can differ from Gaia DR2 proper motion measurements by up to 5 mas $\mathrm{yr}^{-1}$. If we simply fix the time baseline to be 12 years, which is roughly the average time difference between HSC and SDSS DR7, our measurements can differ from Gaia DR2 by up to about 1 mas $\mathrm{yr}^{-1}$.

\section{APPENDIX C: MEASUREMENT ERRORS IN THE PROPER MOTION MEASUREMENTS}

In this appendix we quantify the measurement errors in our proper motion measurements. As indicated, e.g. by the bottom panel of Figure 7, the measurement (statistical) errors are dominant over the proper motion signal on individual star basis. Naively we expect that the measurement errors are mainly from the uncertainties in 


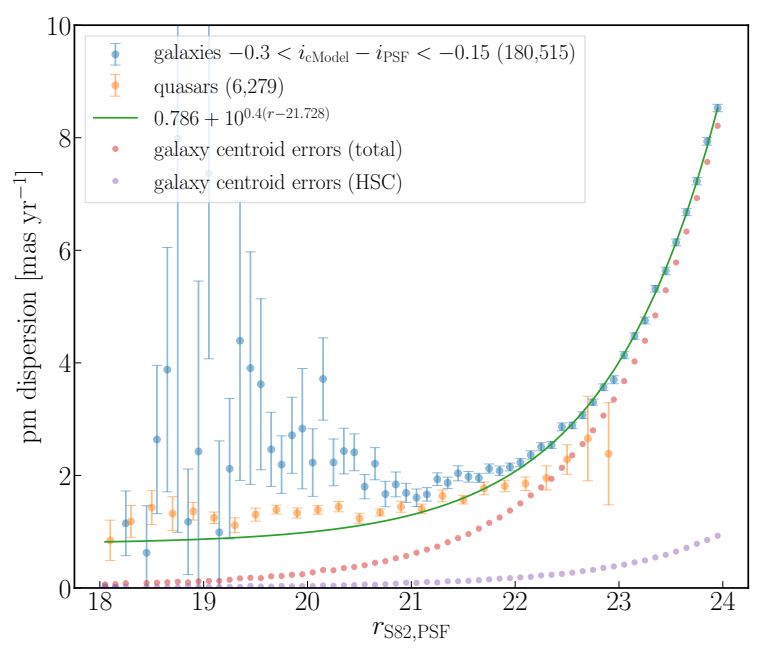

Figure C1. Measurement errors in our proper motion measurements. Blue points are the errors estimated from the dispersion or width of the apparent proper motion distribution of "small-size" galaxies in each bin of the SDSS $r$-band magnitudes, where small-size galaxies are defined from the HSC data based on the criterion $-0.3<i_{\mathrm{HSC}, \mathrm{cModel}}-i_{\mathrm{HSC}, \mathrm{PSF}}<-0.15$ (see text for details). We have 180,515 small-size galaxies in the HSC-S82 regions, and the errorbars are estimated from the bootstrap method. The orange points are the errors estimated from the quasars that are from the same sample in Figure D5. The green line shows the expectation if the errors scale with a flux of object as $1 /$ flux $\propto 10^{0.4(r-21.728)}$, where we find the normalisation and the pivot magnitude $(r=21.728)$ so that the line has a good match to the galaxy results at faint magnitudes. The data points of galaxies nicely follow the expectation. The red points are the errors that are estimated from the quadrature sum of the centroid errors of galaxies in the S82 and HSC catalogues, while the purple points are from the centroid errors in the HSC catalogue alone.

centroid determinations of stars, as reported in the HSC and S82 catalogues, but it turns out that the centroid errors underestimate the measurement errors as we show below. In addition, the measurement errors are from combinations of many effects in our proper motion measurements; photometry, centroid determination, astrometry calibrations, spatial variations of the data quality and calibrations, and so on. Hence it is better to estimate the errors from data themselves.

Since galaxies and quasars do not have proper motions, we can estimate the measurement errors from the dispersion or width in the distribution of apparent proper motions for galaxies/quasars (angular offsets between the HSC and S82 catalogue). However, this is not so easy. For quasars we suffer from the low statistics due to a small size of the sample. On the other hand, galaxies are extended objects, and the proper motion measurements receive additional scatters from uncertainties in the centroid determination due to the finite size. To overcome this obstacle, we choose to use "smallsize" galaxies. We select such small-size galaxies from galaxies satisfying $-0.3<i_{\mathrm{HSC}, \mathrm{cModel}}-i_{\mathrm{HSC}, \mathrm{PSF}}<-0.15$ in Figure 1 . Here the upper cutoff of -0.15 is more conservative than our nominal cut of star/galaxy separation $(-0.08)$ to have a less contamination of stars. The lower cutoff of -0.30 is to remove large-size galaxies. In fact, even if we change these lower and upper cutoffs within the range $-0.20<i_{\mathrm{HSC}, \mathrm{cModel}}-i_{\mathrm{HSC}, \mathrm{PSF}}<-0.30$, the following results remain almost unchanged. Note that we here take advantage of superb image quality of HSC data to identify small-size galaxies. The astrometry of these small-size galaxies has been recalibrated in the same way as for stars, using the recalibration map constructed from all galaxies (see Section 3.3). This removes a contribution of the spatial variations of astrometric systematic effects (the middle panel of Figure 5) to the scatters in the apparent proper motions.

Figure $\mathrm{C} 1$ shows the measurement errors estimated from the scatters in the proper motion measurements for the small-size galaxies in each bin of the S82 magnitudes, which are estimated from the 25 and 75 percentiles of the proper motion distribution in each bin; $\sigma=0.714\left(q_{75}-q_{25}\right)$. We divide the galaxies into bins of the SDSS $r$-band magnitudes because the measurement errors are mainly from the SDSS data, not the HSC data. The errorbars are estimated through bootstrap resampling using 200 realisations in each bin. The orange data points show the results for quasars, which are reasonably similar to the galaxy results at magnitude bins down to $r \simeq 22$, with a small offset. The offset indicates the effect of galaxy extendedness; the galaxy size larger than the PSF increases scatters in the centroid determination. The error curve for quasars at $r \gtrsim 21.5$ are flatter than those of galaxies, but the number of faint quasars in these bins is very small. The green solid line shows the theoretical expectation; if the accuracy of the centroid measurements is limited mainly by the photometry accuracy, the errors scale with fluxes of objects as $1 /$ flux $\propto 10^{r-21.729}$, where $r=21.729$ is the best-fit pivot point to the galaxy results at faint magnitudes (also see Eq. 1 in Bond et al. 2010, for the similar discussion). Our estimation has a good match to the expectation on $r \gtrsim 22$. On the other hand, the red points are the errors estimated from the centroid errors of individual galaxies, quoted from the HSC and S82 catalogues (however, the HSC errors are negligible as shown by the purple dots). The errors in the catalogues underestimate the measurement errors over all the ranges of magnitudes.

Our measurement errors are taken from those of quasars at $r<22$, and from those of small-size galaxies at $\geq 22$. For each star given its $r$-band apparent PSF magnitude from S82, we can estimate its measurement error, $\epsilon_{i}$, according to Figure C1. For a group of $N$ stars, the measurement error of their velocity dispersion is estimated as $\frac{1}{N} \sum_{i=1}^{N} \epsilon_{i}^{2}$.

\section{APPENDIX D: CORRECTION OF THE DCR EFFECT}

\section{D1 DCR correction using the matched stars with Gaia}

As we stressed in the main text, a correction of the DCR effect is

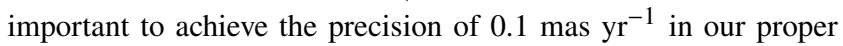
motion measurements, which is important to study the proper motions at large distances $>10 \mathrm{kpc}$. As we will show below we find that the centroid positions of S82 objects are affected by the DCR effect. Here we describe our correction method using the matched stars with Gaia.

Figure D1 shows the comparison against Gaia DR2 proper motion measurements, before correcting for the DCR effect. Note that here we have applied the recalibration of astrometry using galaxies shown in Figure 5. The overall agreement looks very good, with the precision of 0.05 mas $\mathrm{yr}^{-1}$. However, we found that the agreement is a coincidence to some extent, after cancellation of the DCR effects for red and blue stars as we describe below.

We identified subtle systematic differences as follows. If we repeat the comparison against Gaia for red and blue stars separately, using the colour division of $g-r=0.6$, we find discrepancies for each sample from Gaia as demonstrated in Figure D2. The figure shows systematic offsets in the proper motion along the Dec. direction for both red and blue stars, which are almost constant across the R.A. regions. The median offsets averaged over the R.A. 

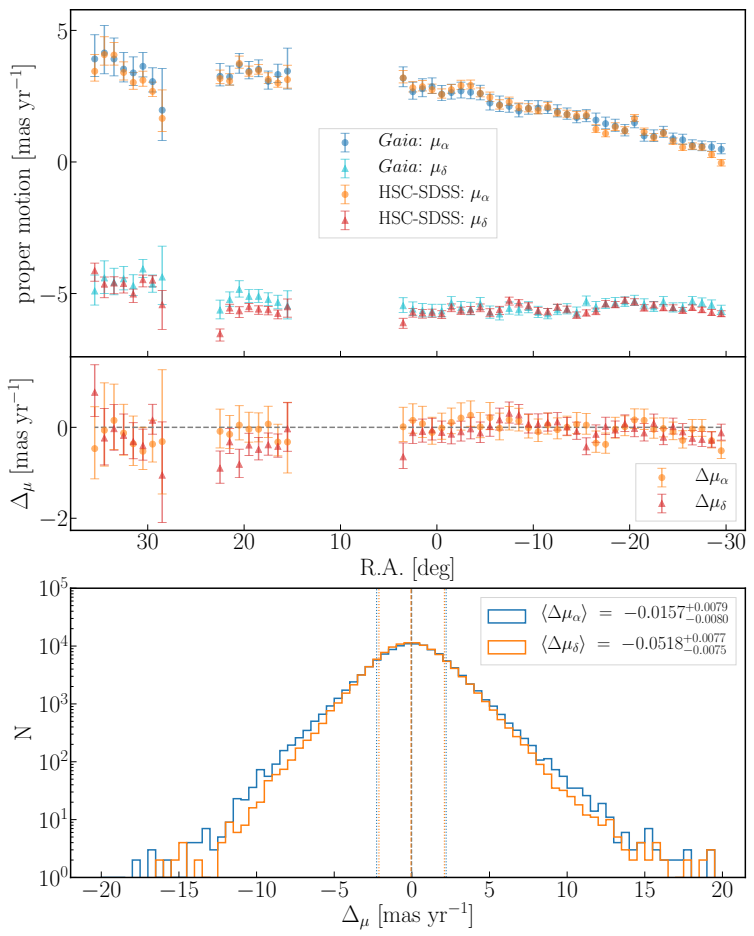

Figure D1. Similar to Figure 7 in the main text, but shows the comparison with Gaia DR2 proper motions before correcting the DCR effect. The median difference of $\mu_{\alpha}$ is $-0.0157 \mathrm{mas} \mathrm{yr}^{-1}$ while that of $\mu_{\delta}$ is $-0.0518 \mathrm{mas} \mathrm{yr}^{-1}$, marked by the vertical dashed lines. The median difference is about 50 times larger than those in Figure 7.

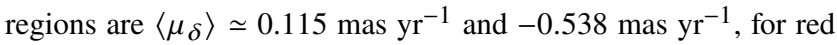
and blue stars, respectively. On the other hand, the measurement of $\mu_{\alpha}$ is consistent with Gaia. That is, there is no sign of residual systematic error in the R.A. direction.

The systematic effects in Figure D2 are consistent with the DCR effect. The SDSS camera does not have such a DCR corrector. DCR stretches an observed image of source along the zenith direction due to the wavelength-dependent refraction by atmosphere and, as a result, each object appears to be observed at a shifted position in the camera image by a different amount in different filters. For SDSS, the zenith direction is mainly along the Dec. direction for the Stripe 82 region, which is consistent with our findings. Since the spectral energy distribution (SED) of galaxies and stars are different, our astrometry recalibration method using the galaxy positions (Figure 5) cannot fully correct for the DCR effects for stars.

Figure D3 further examines how the DCR effects depend on colours of stars. Thanks to the large sample of stars even after subdivision based on the colour, the comparison with Gaia clearly shows that the DCR effects vary depending on colours of stars relative to those of galaxies. The figure shows there is almost no DCR effect in the R.A. direction. The $(g-i)$ colour in Figure D3 displays a wider dynamic range for the DCR effect compared to the $(g-r)$ colour, because very red stars would have very similar colour in $g-r(g-r \sim 1.6)$, but still have different colours in $g-i$. For blue stars with $g-r<0.6$, which roughly corresponds to $g-i<0.6$ as well, the averaged DCR offset is consistent with the amount of difference from Gaia in Figure D2. The smaller amount of difference from Gaia for red and all stars, compared that for blue stars, is a result of averaging both positive and negative DCR effects in Figure D3.
We use the measured offsets in Dec. direction, $\Delta \mu_{\delta}$, against the $(g-i)$ colour to infer the DCR effect for the matched stars with Gaia. Then, for a given main-sequence star in our main analysis, we infer the DCR effect from the $(g-i)$ colour of the star, obtained by linearly interpolating the result of Figure D3, and then correct for the DCR effect for the star. This is our default analysis we use in this paper. Figure 7 shows the results after including the DCR correction. The median differences from Gaia is reduced by con-

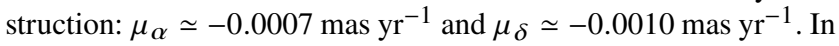
particular, comparing Figures 7 and D1 manifests that the discrepancy from Gaia at R.A. 20 deg. is significantly improved by our DCR correction method.

\section{D2 Quasar validation of the DCR correction method}

As we described in the preceding subsection, the DCR effect is important to take into account. In this subsection we use quasars to make an independent confirmation of the DCR effect and also discuss the robustness of astrometry recalibration in Section 3.3.

We adopt spectroscopic quasars from SDSS DR 14 to ensure the sample purity. We match their positions in DR14 to those matched HSC-S82 objects via the source positions from S82. Since the images of most DR14 quasars are taken before DR7, we do not expect their astrometric positions to be very different from those of S82. We adopt a small angular separation of $0.2^{\prime \prime}$ for the matching. In the end, 8,757 quasars are matched after a 3- $\sigma$ clipping over the angular offsets between HSC and S82 (see Table 1).

We show the median angular offset per year in each R.A. bin for both quasars and galaxies in the upper two panels of Figure D4. Note this is before applying any astrometry recalibrations using galaxies (see Section 3.3 for more details). The figure shows a nearly constant offset in the Dec. direction, without strong R.A. dependence, while the offset in R.A. direction is consistent with no offset (zero).

The lower panel shows the histogram distributions of angular offsets of quasars, after applying the astrometry recalibration using galaxies, i.e., the same method as how we recalibrate the astrometry for stars, but we did not include any correction for DCR yet. Since distant quasars do not move, any observed proper motions reflect residual systematics in our astrometry recalibration. There is almost no bias in R.A., while it shows a non-negligible bias in the Dec.

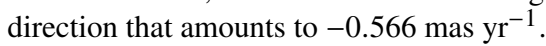

The level of residual systematics is similar to the difference between quasars and galaxies shown in the top and middle panels of Figure D4, and is in good agreement with the median difference against Gaia DR2 proper motions for blue stars in Figure D2, which is caused by the effect of DCR. Quasars are quite blue objects suffering more from DCR while galaxies can have very different SED compared with quasars. Because we use galaxies to recalibrate the astrometry for quasars, the difference in quasar and galaxy positions due to DCR remains in the recalibrated results, revealing it as residual systematic errors.

The reference bands for astrometric measurements are $r$-band and $i$-band for S 82 and HSC, respectively. We repeat the comparison between quasars and galaxies for the positions in other SDSS filters. We find the difference between quasars and galaxies is larger in $g$ -

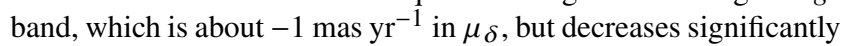
in $r$ and $i$-bands. This is consistent with the expected effect caused by DCR, which is more severe in blue bands. With these results we conclude that the discrepancy between quasars and galaxies is resulted from the effect of DCR.

In Figure D5 we further validate the DCR effect for quasars. Here, as we did for the main results, we apply the DCR correction 

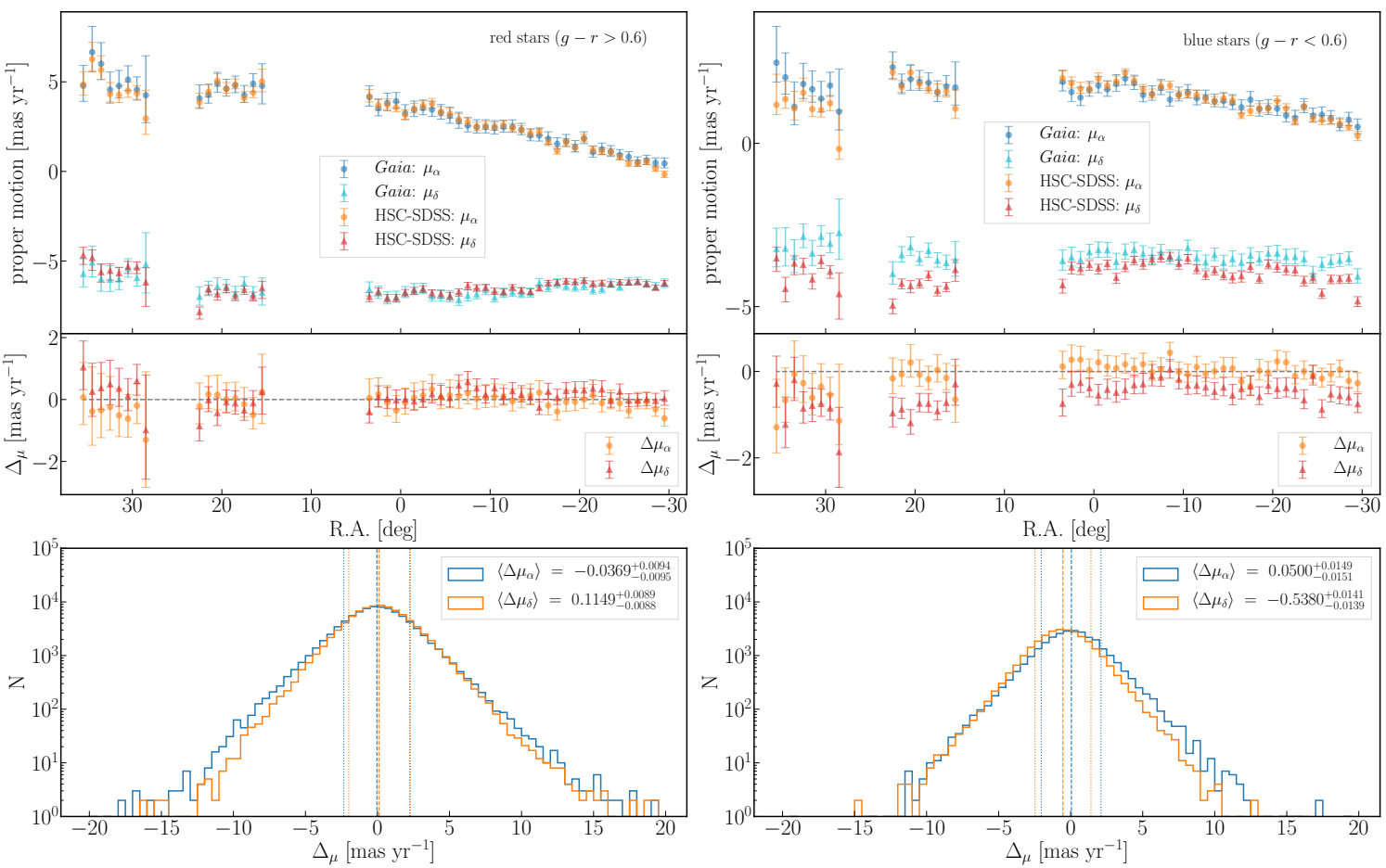

Figure D2. Similar to Figure 7, but the plot shows the comparison of our proper motion measurements against the Gaia DR2 proper motions for red (left) and blue (right) stars, respectively, that are divided by the colour cut of $g-r=0.6$.
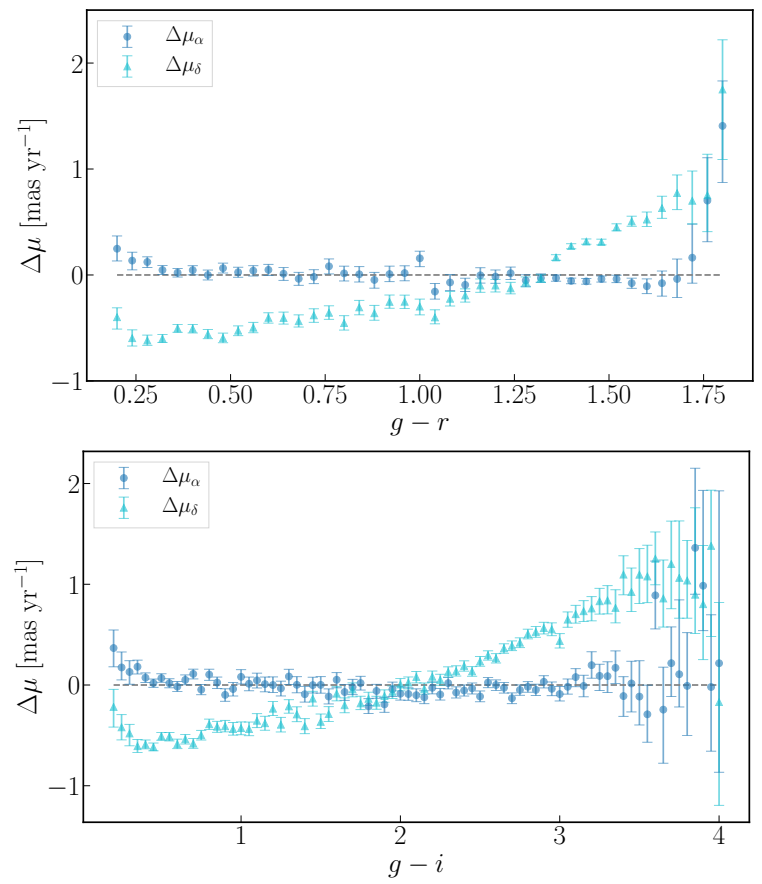

Figure D3. The proper motion differences between the HSC-S82 and Gaia for stars as a function of the colours $(g-r)$ (upper panel) or $(g-i)$ (lower), where we estimate the averaged proper motion difference over the survey footprints for subsamples of stars divided in each bin of the colour ( $x$-axis). Note that samples of blue and red stars we use in our main analysis are defined by the colour cut, $g-r<0.6$ or $>0.6$, respectively. to individual quasars based on their colour $(g-i)$ in Figure D3. If the angular offsets of quasars in Figure D4 are due to the DCR effect, we expect that our method can correct for the angular offsets, because the DCR effects depend primarily on colours of objects (even if the SEDs of quasars are different from those of the Gaiamatched stars used to construct the DCR correction template). We have 6,288 quasars in the range of the $(g-i)$ colour in Figure D3 in the HSC-S82 region. Figure D5 shows that our method remarkably well corrects for the angular offsets of quasars to the accuracy of less than 0.1 mas $\mathrm{yr}^{-1}$ in both R.A. and Dec. directions. With these results, we conclude that the primary systematic errors in our astrometric solutions are the DCR effect, and our correction method has a very good control of the DCR effect by less than 0.1 mas $^{-1} r^{-1}$.

This paper has been typeset from a $\mathrm{TE}_{\mathrm{E}} \mathrm{X} / \mathrm{L} \mathrm{T} \mathrm{E}$ file prepared by the author. 

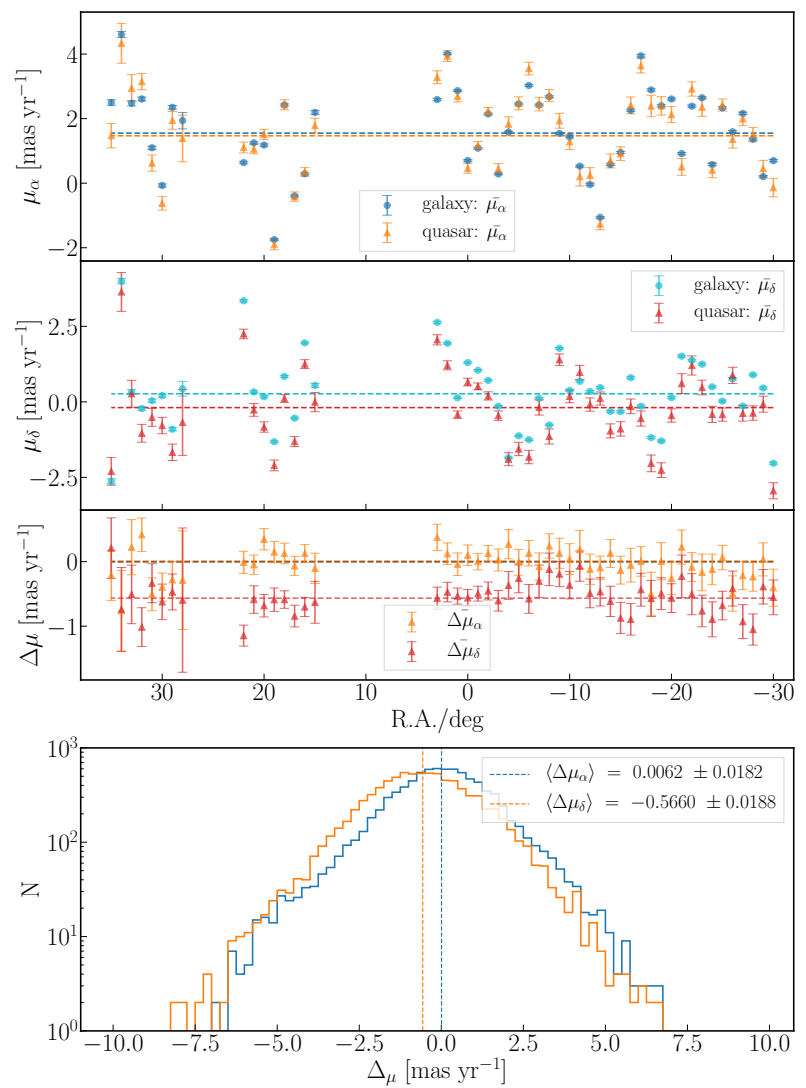

Figure D4. Angular offsets in R.A. and Dec. directions, in units of [ $\mathrm{mas}_{\mathrm{yr}}{ }^{-1}$ ] as in Figure 5, measured from the galaxies and quasars in the HSC and S82 catalogues (see Table 1) in each R.A. range ( $x$-axis). Horizontal lines with the corresponding colour denote the level of systematics averaged over all bins of R.A.. The middle panel, with $\Delta \mu$ in the $y$-axis label, denotes the differences between the angular offsets of galaxies and quasars in each R.A. bin. There is a clear offset in the Dec. direction, as expected by the effect of differential chromatic diffraction (DCR) (see text for details). The lower panel shows the distribution of the differences between the angular offsets for individual quasars between $\mathrm{HSC}$ and S82, after performing the recalibration of astrometric solutions using the galaxy offsets as in Figure 5. There is a residual systematic error in the Dec. direction, given by -0.5660 mas yr $^{-1}$ that is significant compared to the error (more than $2 \sigma$ ).
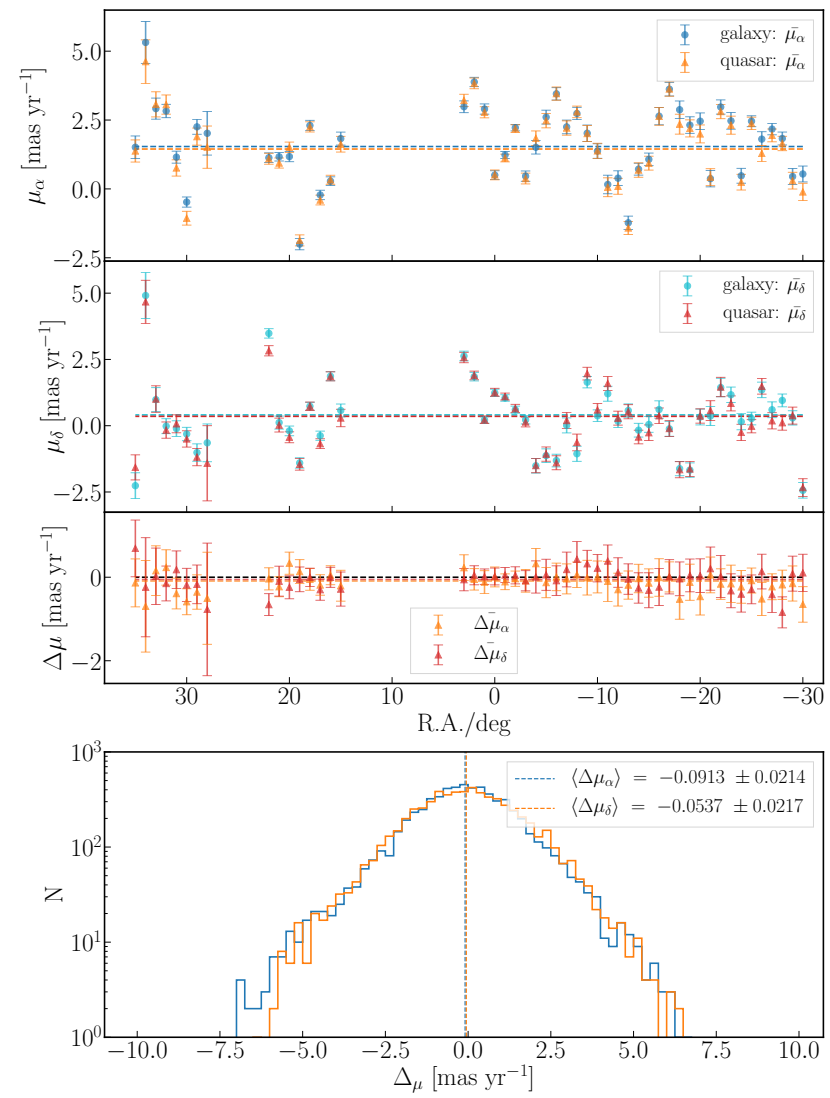

Figure D5. Validation of the DCR correction method using the quasars. Here we apply the DCR correction method to individual quasars based on their HSC colour $(g-i)$; we infer each quasar's colour in $g-i$ to infer the DCR effects by matching the colour to the DCR- $(g-i)$ colour for the Gaia matched stars in Figure D3. For the range of $(g-i)$ in Figure D3, we have 6,288 quasars in the HSC-S82 region. The three panels are similar to the previous figure. The upper and lower panels show the angular offsets between the HSC and S82 catalogues for the galaxies and the quasars in R.A. and Dec. directions, after implementing the DCR correction. The lower panel show the distribution, and the numbers in the legend give the averaged differences between the angular offsets. In doing this, we used galaxies in the grids of the calibration map in Figure 5 where the quasars in the sample exist. 